\title{
VLT/UVES spectroscopy of individual stars in three globular clusters in the Fornax dwarf spheroidal galaxy $\star, \star \star$
}

\author{
B. Letarte ${ }^{1}$, V. Hill ${ }^{2}$, P. Jablonka ${ }^{3,4}$, E. Tolstoy ${ }^{1}$, P. François ${ }^{2}$, and G. Meylan ${ }^{4}$ \\ 1 Kapteyn Astronomical Institute, University of Groningen, PO Box 800, 9700AV Groningen, The Netherlands \\ e-mail: [bruno; etolstoy] @astro.rug.nl \\ 2 Observatoire de Paris, GEPI CNRS UMR 8111, 2 Pl. Jules Janssen, 92195 Meudon Cedex, France \\ e-mail: [vanessa.hill;patrick.francois]@obspm.fr \\ 3 Observatoire de Genève, 1290 Sauverny, Switzerland \\ e-mail: pascale.jablonka@obs.unige.ch \\ ${ }^{4}$ Laboratoire d'Astrophysique de l'École Polytechnique Fédérale de Lausanne (EPFL), 1290 Sauverny, Switzerland \\ e-mail: georges.meylan@epfl.ch
}

Received 28 October 2005 / Accepted 12 March 2006

\section{ABSTRACT}

\begin{abstract}
We present a high resolution $(R \sim 43000)$ abundance analysis of a total of nine stars in three of the five globular clusters associated with the nearby Fornax dwarf spheroidal galaxy. These three clusters (1,2 and 3) trace the oldest, most metal-poor stellar populations in Fornax. We determine abundances of $\mathrm{O}, \mathrm{Mg}, \mathrm{Ca}, \mathrm{Ti}, \mathrm{Cr}, \mathrm{Mn}, \mathrm{Fe}, \mathrm{Ni}, \mathrm{Zn}, \mathrm{Y}, \mathrm{Ba}, \mathrm{Nd}$ and $\mathrm{Eu}$ in most of these stars, and for some stars also $\mathrm{Mn}$ and $\mathrm{La}$. We demonstrate that classical indirect methods (isochrone fitting and integrated spectra) of metallicity determination lead to values of $[\mathrm{Fe} / \mathrm{H}]$ which are 0.3 to $0.5 \mathrm{dex}$ too high, and that this is primarily due to the underlying reference calibration typically used by these studies. We show that Cluster 1 , with $[\mathrm{Fe} / \mathrm{H}]=-2.5$, now holds the record for the lowest metallicity globular cluster. We also measure an over-abundance of Eu in Cluster 3 stars that has only been previously detected in a subgroup of stars in M 15 . We find that the Fornax globular cluster properties are a global match to what is found in their Galactic counterparts; including deep mixing abundance patterns in two stars. We conclude that at the epoch of formation of globular clusters both the Milky Way and the Fornax dwarf spheroidal galaxy shared the same initial conditions, presumably pre-enriched by the same processes, with identical nucleosynthesis patterns.
\end{abstract}

Key words. stars: abundances - galaxies: dwarf - galaxies: star clusters

\section{Introduction}

It is now established that some dwarf galaxies have globular cluster systems around them (Lotz et al. 2004; van den Bergh 2005; Seth et al. 2004). Their possible common origin with clusters in larger parent galaxies, the link between the dwarf galaxy field and globular cluster stars are open questions to be addressed. The largest samples of dwarf galaxies with globular cluster systems are however distant, and this restricts the analyses to using integrated properties.

Fornax and Sagittarius are the nearest dwarf spheroidal galaxies (dSph) with globular clusters and can be resolved into individual stars. The Fornax dSph contains five star clusters (Shapley 1938; Hodge 1961) and while the Sagittarius dSph is obscured by dust and confused by merging with our Galaxy, Fornax is high above the Galactic plane, therefore offering a uniquely useful target for investigation, see Fig. 1.

The ages of the Fornax globular clusters have been determined by fitting isochrones to deep HST Colour-Magnitude Diagrams [CMDs] (Buonanno et al. 1998, 1999). They are found to be the same age as old metal-poor Galactic clusters M 92 and M 68 (around 13 Gyr old) to within \pm 1 Gyr, with the

^ Based on UVES observations collected at the European Southern Observatory, proposal number 70.B-0775.

$\star \star$ Appendices are only available in electronic form at http://www.edpsciences.org

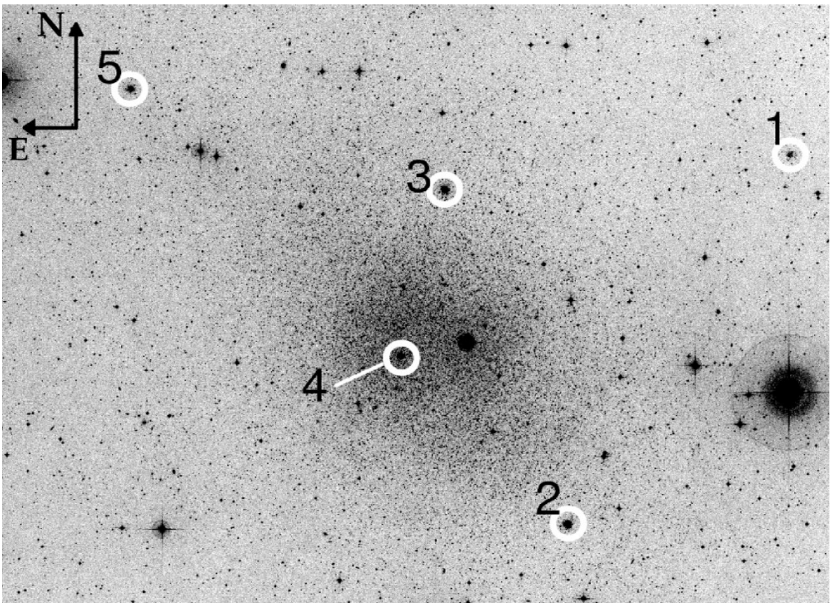

Fig. 1. $A \approx 85^{\prime} \times 62^{\prime}$ DSS image of the Fornax dSph. North is up and East is to the left, as indicated. We have marked the position of the 5 GCs using the numbering scheme defined by Shapley (1938) and Hodge (1961).

exception of Cluster 4, which seems buried in the center of Fornax and maybe younger by about 3 Gyr. The cluster metallicities have been estimated with different techniques ranging from fitting a slope to the Red Giant Branch (RGB) to high and medium resolution spectroscopy of the integrated light of 
Table 1. Observation Log.

\begin{tabular}{|c|c|c|c|c|c|c|c|}
\hline Our id & Litterature IDs & $\begin{array}{r}\text { RA }(\mathrm{J} 2000) \\
\text { degrees }\end{array}$ & $\begin{array}{r}\text { Dec }(\mathrm{J} 2000) \\
\text { degrees }\end{array}$ & $\begin{array}{r}\text { Exp. time } \\
\text { (s) }\end{array}$ & $\begin{array}{l}/ N @ \\
670 \mathrm{~nm}\end{array}$ & $\begin{array}{r}V_{\text {rad }} \\
\left(\mathrm{km} \mathrm{s}^{-1}\right)\end{array}$ & Comments \\
\hline Cl1-D56 & $\mathrm{D} 56^{b}, \mathrm{~J} 24^{c}$ & 39.254958 & -33.17790 & 14400 & 23 & 57.6 & same slit as D68 \\
\hline Cl1-D68 & $\mathrm{B} 51^{a}, \mathrm{D} 68^{b}, \mathrm{~J} 23^{c}$ & 39.254609 & -34.17875 & 14400 & 50 & 60.2 & same slit as D56 \\
\hline Cl1-D164 & $\mathrm{B} 18^{a}, \mathrm{D} 164^{b}, \mathrm{~J} 65^{c}, \mathrm{~B} 713^{d}$ & 39.257554 & -34.18628 & 18000 & 30 & 60.0 & \\
\hline $\mathrm{Cl} 2-\mathrm{B} 71$ & $\mathrm{~B} 71^{a}$ & 39.677388 & -34.80412 & 21600 & 30 & 63.4 & \\
\hline Cl2-B74 & $\mathrm{B} 74^{a}$ & 39.684917 & -34.80301 & 14400 & $\ldots$ & & Too faint \\
\hline $\mathrm{Cl2}-\mathrm{B} 77$ & $\mathrm{~B} 77^{a}$ & 39.685203 & -34.80303 & 14400 & 30 & 64.1 & same slit as B74 \\
\hline $\mathrm{Cl} 2-\mathrm{B} 200$ & $\mathrm{~B} 200^{d}$ & 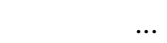 & $\ldots$ & 3900 & $\ldots$ & $\ldots$ & carbon star \\
\hline $\mathrm{Cl} 2-\mathrm{B} 226$ & $\mathrm{~B} 226^{d}$ & 39.682143 & -34.80801 & 7200 & 40 & 64.0 & \\
\hline Cl3-B59 & $\mathrm{B} 59^{a}, \mathrm{~J} 9^{c}$ & 39.942803 & -34.25855 & 10800 & 30 & 59.7 & \\
\hline Cl3-B61 & $\mathrm{B} 61^{a}, \mathrm{~J} 31^{c}$ & 39.957271 & -34.25782 & 21600 & 30 & 63.7 & \\
\hline $\mathrm{Cl} 3-\mathrm{B} 82$ & $\mathrm{~B} 82^{a}, \mathrm{~J}^{c}$ & 39.951057 & -34.25277 & 14400 & 40 & 64.8 & \\
\hline M15-S1 & $\mathrm{S} 1^{e}, \mathrm{~K} 431^{f}$ & 322.484344 & 12.21002 & 900 & 113 & -106.4 & \\
\hline M15-S3 & $\mathrm{S} 3^{e}, \mathrm{~K} 387^{f}$ & 322.481920 & 12.21231 & 1200 & 115 & -111.3 & \\
\hline M15-S4 & $\mathrm{S} 4^{e}, \mathrm{~K} 825^{f}$ & 322.509599 & 12.18986 & 750 & 122 & -101.4 & spec. double star \\
\hline M15-S6 & $\mathrm{S} 6^{e}, \mathrm{~K} 1040^{f}$ & 322.543661 & 12.16832 & 1000 & 96 & -100.0 & \\
\hline M15-S7 & $\mathrm{S} 7^{e}, \mathrm{~K} 146^{f}$ & 322.457798 & 12.13489 & 900 & 83 & -100.7 & \\
\hline
\end{tabular}

$\mathrm{ID}^{a}$ from Buonanno et al. (1985); $\mathrm{ID}^{b}$ from Demers et al. (1990); ID ${ }^{c}$ from Jorgensen \& Jimenez (1997); ID ${ }^{d}$ from Buonanno et al. (1998); ID $^{e}$ from Sandage (1970); ID $^{f}$ from Kustner (1921).

the cluster. Conclusions vary from one work to another, as summarized in Strader et al. (2003), but the clusters definitely appear more metal-poor than the bulk of the galaxy field stellar population, with bluer RGBs, well populated blue horizontal branches (HB) and a range of HB morphology (Buonanno et al. 1998, 1999). Saviane et al. (2000) showed that the Fornax $\mathrm{dSph}$ field star colour distribution is well fitted by two Gaussian functions, best interpreted as a bi-modal metallicity distribution, with the older population having a wide abundance range between -2.2 and -1.4 . Stars as young as $10^{8} \mathrm{Myr}$ have also been discovered in the field of Fornax (Stetson et al. 1998). In this framework, the globular clusters of Fornax dSph trace the first stages of star formation in the galaxy.

High resolution spectroscopy of individual stars in the clusters is the only way to assess the abundances of individual chemical species. Alpha, iron-peak, heavy -elements provide essential clues on (i) the conditions of formation of the globular clusters in a dwarf galaxy, including epoch and time scales (ii) to probe the nucleosynthesis in a galactic system with a star formation history that is fundamentally different from that of the Milky Way. We present here a VLT/UVES spectroscopic analysis of a total sample of nine stars in three Fornax dSph clusters.

\section{Observations}

We targeted Cluster 1, Cluster 2 and Cluster 3, to span the Fornax globular cluster system range of distances from the galaxy centre avoiding regions of heavy crowding. We also sampled a range of HB morphology, as well as the metallicity and concentration ranges. Cluster 1, at a radial distance of $43 \mathrm{arcmin}$ (or $1.75 \mathrm{kpc}$ at the distance of Fornax dSph) from the galaxy center, is diffuse, with low surface brightness, most of its HB is red. Cluster 2, located at 25 arcmin $(1 \mathrm{kpc})$ from the galaxy center, is slightly more concentrated and exhibits a more extended HB. Finally, Cluster 3 at a galactocentric radial distance of $13 \operatorname{arcmin}(530 \mathrm{pc})$ is very dense and has an extended HB.

We used the red arm of UT2/UVES, CD\#3, centered at $580 \mathrm{~nm}$, with a wavelength range of 480-680 nm (Dekker et al. 2000 ) in visitor mode in October 2002. We obtained spectra with a resolution of $\sim 43000$ and average $S / N \sim 20-30$ per pixel with an integration time of $2-6 \mathrm{~h}$ for each of the nine individual stars in Fornax dSph globular Clusters 1, 2 and 3. The stars were selected to be on the RGB from CMDs, (Buonanno et al. 1985; Demers et al. 1990; Jorgensen \& Jimenez 1997; Buonanno et al. 1998). Their individual finding charts are shown in Fig. 2. We also observed 5 calibration red giant branch stars in the well studied globular cluster M 15 (Sneden et al. 1997). The observations of M 15 stars provide an independent check on our data reduction and analysis methods. Details of the observations are shown in Table 1, including the derived radial velocities $V_{\text {rad }}$ and $S / N$ ratios.

\section{Data reduction and analysis}

The spectra were extracted with the standard UVES pipeline, except for two pairs of stars on the same slit which we had to reduce interactively using the UVES context within MIDAS (see Table 1). At the telescope we already identified Cl2-B200 as a carbon star, and it was discarded from further analysis. As already noted by Sneden et al. (1997), M 15-S4 is probably a spectroscopic double star, as all lines are significantly wider (larger Full Width Half Maximum [FWHM]) than the other stars of M 15. It was not used for our abundance analysis.

For each of our targets we made equivalent width $(E W)$ measurements with SPLOT in IRAF, except for the lines with a small $E W(\$ 50 \mathrm{~m} \AA)$. For these weak lines, we noticed that SPLOT was giving very unstable FWHM measurements. A home-made gaussian-fitting program was used for these lines to fix the FWHM at the instrumental value. We also used DAOSPEC ${ }^{1}$, a new programme that automatically measures $E W \mathrm{~s}$ by iteratively fitting gaussians of fixed FWHM to all lines in the spectrum and removing the continuum signature (Stetson \& Pancino, in preparation). Having confirmed that DAOSPEC gives results compatible with those obtained by hand for lines of moderate strength $(E W \leq 150 \mathrm{~m} \AA)^{2}$, we used DAOSPEC measured $E W \mathrm{~s}$ for M 15.

\footnotetext{
1 http://cadcwww . dao.nrc.ca/stetson/daospec/.

2 We note here for completeness that, at this high resolution, the fixed FWHM Gaussian hypothesis adopted by DAOSPEC does not hold for the strongest lines $(E W>150 \mathrm{~m} \AA)$ where departures from the Gaussianity and natural broadening play a significant role.
} 


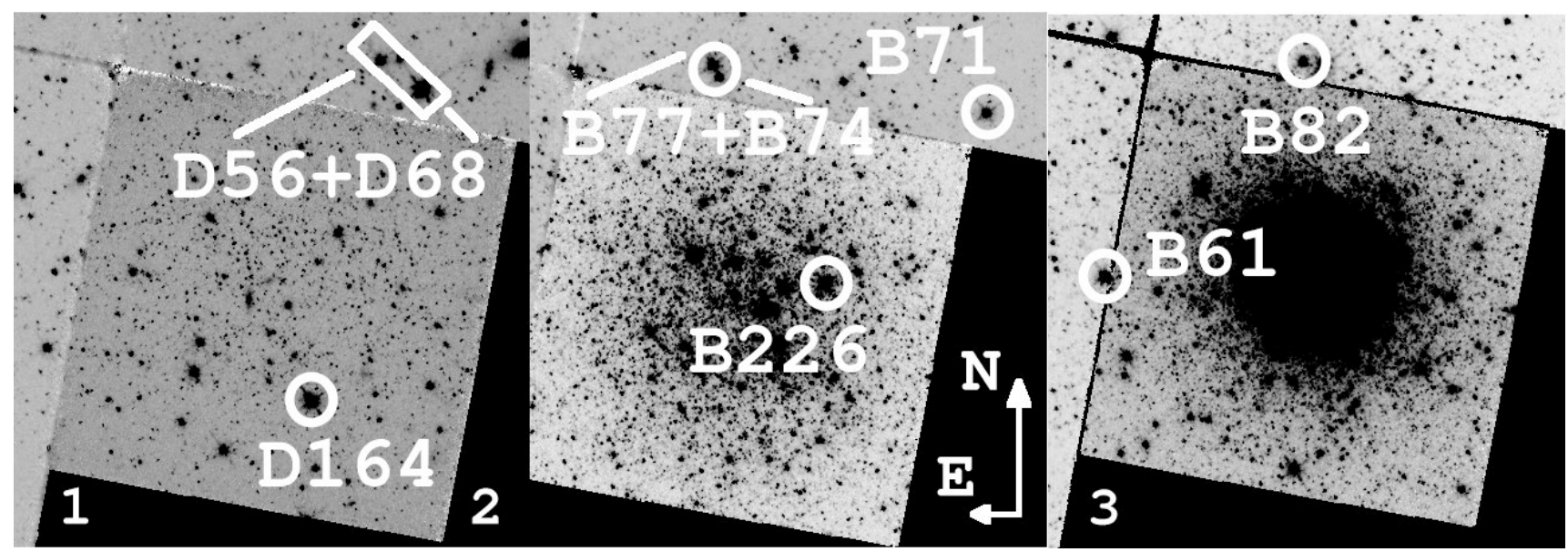

Fig. 2. The finding charts for our observations of the Fornax GCs, from 1 (left) to 3 (right). North is up and East is left, as indicated. Note that star Cl3-B59 is outside of the cluster 3 HST field, to the west.

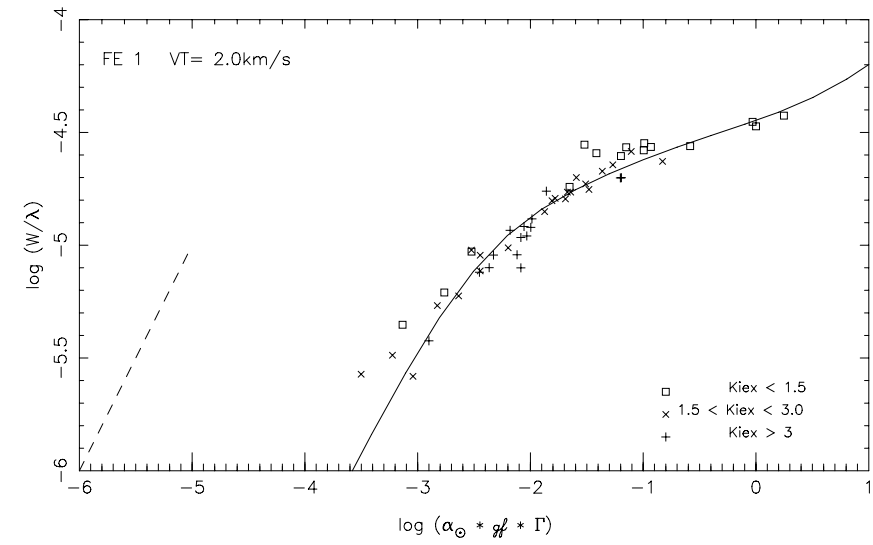

Fig. 3. Observed curve of growth for $\mathrm{Fe} \mathrm{I}$ in $\mathrm{Cl} 3-\mathrm{B} 82$. The dotted line marks the $[\mathrm{Fe} / \mathrm{H}]=0$ location, while the full line is the theoretical curve of growth for a typical $\mathrm{Fe}$ I line with the stellar parameters adopted for this star.

We can detect a range of elements in our coadded spectra: Fe I, Fe II, Ti I, Ti II, O I, Mg I, Ca I, Cr I, Mn I, Ni I, Zn II, Y II, Ba II, La II, Nd II and Eu II, which allows us to achieve a comprehensive abundance analysis. The most important is Fe, with an average of 50 measured lines for Fe I and 10 lines for Fe II. Line parameters and $E W$ measurements for all stars are reported in Table A.1. Abundances for the different elements were calculated with CALRAI, originally described in Spite (1967) with many improvements over the years. The stellar atmospheres models are those of Plez (private communications, 2000, 2002; described in Cayrel et al. 2004). Spectral synthesis was required for some elements: $\mathrm{Eu}, \mathrm{Zn}, \mathrm{Mg}, \mathrm{Na}, \mathrm{O}$ and $\mathrm{Ba}$ to account for hyperfine splitting (Eu, Ba); weak lines $(\mathrm{Zn}, \mathrm{O})$ and strong, possibility saturated lines ( $\mathrm{Na}, \mathrm{Mg}$ ).

Initial guesses were made for the stellar effective temperature $\left(T_{\text {eff }}\right)$ using $V-I$ and/or $B-V$ colours, using the Alonso et al. (2001) calibration and a reddening of $E(B-V)=0.065$. The surface gravity $(\log g)$ was estimated assuming a $0.8 M_{\odot}$ mass for the stars, a distance modulus of $(m-M)=20.85 \mathrm{mag}$ and bolometric corrections from Alonso et al. (2001). However, the quality of the photometric data we gathered for these stars turned out to be too poor to constrain firmly the star's effective temperature (only 2 stars had HST photometry in Buonnano et al. 1998, and the other photometric sources were ground-based, suffering
Table 2. Adopted parameters of the stellar atmosphere model for each star.

\begin{tabular}{lrrrr}
\hline \hline Star ID & $T_{\text {eff }}(\mathrm{K})$ & $\log g$ & {$[\mathrm{Fe} / \mathrm{H}]$} & $V_{\mathrm{t}}\left(\mathrm{km} \mathrm{s}^{-1}\right)$ \\
\hline Cl1-D56 & 4600 & 1.0 & -2.60 & 2.1 \\
Cl1-D68 & 4350 & 0.5 & -2.60 & 2.0 \\
Cl1-D164 & 4400 & 0.8 & -2.60 & 2.1 \\
Cl2-B71 & 4450 & 0.7 & -2.10 & 1.8 \\
Cl2-B77 & 4350 & 0.7 & -2.10 & 1.7 \\
Cl2-B226 & 4250 & 0.6 & -2.10 & 2.0 \\
Cl3-B59 & 4400 & 0.5 & -2.30 & 1.8 \\
Cl3-B82 & 4350 & 0.5 & -2.30 & 2.0 \\
\hline M 15-S1 & 4350 & 0.5 & -2.40 & 1.9 \\
M 15-S3 & 4400 & 0.6 & -2.40 & 1.8 \\
M 15-S4 & 4150 & 0.6 & -2.30 & 2.3 \\
M 15-S6 & 4400 & 0.7 & -2.40 & 1.8 \\
M 15-S7 & 4400 & 0.4 & -2.50 & 1.9 \\
\hline
\end{tabular}

from crowding and not all in a homogeneous photometric system). We therefore chose to base our analysis solely on spectroscopic criteria. The $T_{\text {eff }}, \log g$ and micro-turbulence velocity $\left(V_{\mathrm{t}}\right)$ were adjusted to insure that we had the ionisation balance of Fe I and Fe II and that the Fe I abundance is independent of both line strength and excitation potential of the line. Figure 3 illustrates the quality of our solution by showing the curve of growth obtained for $\mathrm{Cl} 3-\mathrm{B} 82$, where we notice that every part of the curve of growth is well populated. The final set of stellar parameters used for each star are shown in Table 2 . The $[\mathrm{Fe} / \mathrm{H}]$ in this table is the metallicity of the model used to compute the abundances, not the final abundance value of Fe I or Fe II of the star.

As an additional test, since the $S / N$ reached in the individual spectra was rather limited, we also co-added the spectra of stars with similar parameters within each cluster (all three stars of Cluster 3 on the one hand, and the two cooler stars of Cluster 1 on the other hand), and repeated the analysis. The results are fully consistent with the analysis of the individual stars: $T_{\mathrm{eff}}$, $\log g$ and $V_{\mathrm{t}}$ are undistinguishable, while the mean $[\mathrm{Fe} / \mathrm{H}]$ is recovered within 0.02 dex, and most of the other abundance ratios fall well within the star to star scatter.

A significant source of error in our analysis is the uncertainty in measuring the $E W$. Our error in the $E W$ determinations were estimated by propagating the $E W$ error estimates (from splot) through the abundance computation (the abundances $E W+\delta E W$ and $E W-\delta E W$ were computed and compared to the central 


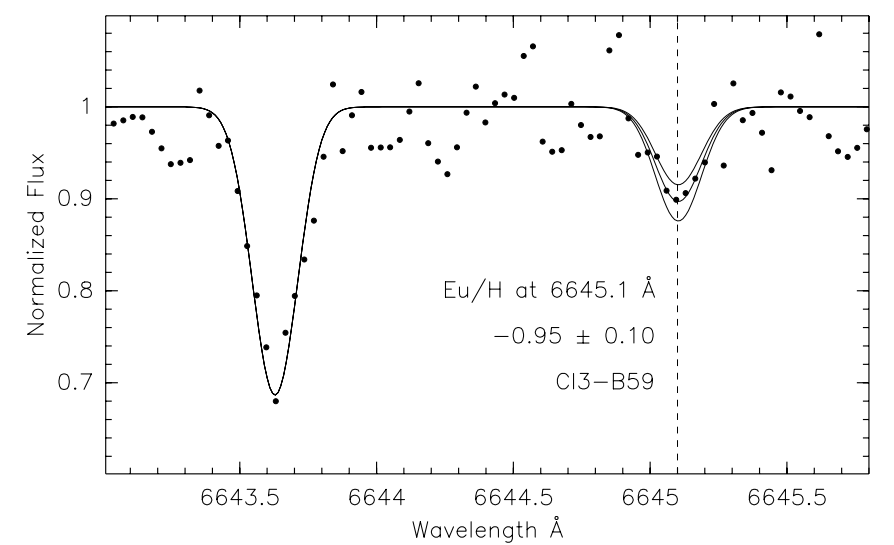

Fig. 4. The synthetic spectra for the Eu line at $\lambda=6645.1 \AA$ overlaid on the data for Cl3-B59. The middle line is the adopted fit, while the lower and upper ones are the error estimate of $\pm 0.1 \mathrm{dex}$. The larger line on the left is a Ni line.

Table 3. Dependencies on model atmosphere parameters.

\begin{tabular}{lrrrr}
\hline \hline & $\Delta T_{\text {eff }}$ & $\Delta \log g$ & $\Delta V_{\mathrm{t}}$ & Combined \\
& $-200 \mathrm{~K}$ & -0.3 & $-0.2 \mathrm{~km} \mathrm{~s}^{-1}$ & \\
\hline$[\mathrm{Ba} \mathrm{II} / \mathrm{Fe} \mathrm{I}]$ & -0.11 & 0.11 & -0.06 & 0.17 \\
{$[\mathrm{Ca} \mathrm{I} / \mathrm{Fe} \mathrm{I}]$} & 0.00 & -0.01 & 0.05 & 0.05 \\
{$[\mathrm{Cr} \mathrm{I} / \mathrm{Fe} \mathrm{I}]$} & 0.21 & -0.03 & -0.02 & 0.21 \\
{$[\mathrm{Eu} \mathrm{II} / \mathrm{Fe} \mathrm{I}]$} & -0.16 & 0.13 & 0.08 & 0.22 \\
{$[\mathrm{Fe} \mathrm{I} / \mathrm{H}]$} & 0.27 & -0.05 & -0.10 & 0.29 \\
{$[\mathrm{Fe} \mathrm{II} / \mathrm{H}]$} & -0.05 & 0.07 & -0.06 & 0.10 \\
{$[\mathrm{La} \mathrm{II} / \mathrm{Fe} \mathrm{I}]$} & -0.12 & 0.12 & 0.08 & 0.19 \\
{$[\mathrm{Mg} \mathrm{I} / \mathrm{Fe} \mathrm{I}]$} & -0.06 & -0.08 & 0.02 & 0.10 \\
{$[\mathrm{Mn} \mathrm{I} / \mathrm{Fe} \mathrm{I}]$} & -0.02 & -0.01 & 0.08 & 0.08 \\
{$[\mathrm{Na} \mathrm{I} / \mathrm{Fe} \mathrm{I}]$} & 0.18 & -0.04 & -0.04 & 0.19 \\
{$[\mathrm{Nd} \mathrm{II} / \mathrm{Fe} \mathrm{I}]$} & -0.14 & 0.10 & 0.04 & 0.18 \\
{$[\mathrm{Ni} \mathrm{I} / \mathrm{Fe} \mathrm{I}]$} & 0.02 & -0.02 & -0.03 & 0.04 \\
{$[\mathrm{O} \mathrm{I} / \mathrm{Fe} \mathrm{I}]$} & -0.11 & 0.13 & 0.08 & 0.19 \\
{$[\mathrm{Ti} / \mathrm{Fe} \mathrm{I}]$} & 0.27 & -0.02 & 0.02 & 0.27 \\
{$[\mathrm{Ti} \mathrm{II} / \mathrm{Fe} \mathrm{I}]$} & -0.21 & 0.09 & 0.02 & 0.23 \\
{$[\mathrm{Y} \mathrm{I} / \mathrm{Fe} \mathrm{I}]$} & -0.18 & 0.09 & 0.04 & 0.21 \\
{$[\mathrm{Zn} \mathrm{I} / \mathrm{Fe} \mathrm{I}]$} & -0.28 & 0.03 & 0.06 & 0.29 \\
\hline
\end{tabular}

adopted value). For elements which were computed by spectral synthesis, the error is estimated by eye, plotting a range of acceptable fits, as illustrated in Fig. 4 for the weak Eu line in $\mathrm{Cl} 3-\mathrm{B} 59$. Another way to estimate the measurement errors affecting the abundance is to consider the dispersion (rms) around the mean. For species with sufficient number of lines measured $(>3)$, this dispersion was adopted whereas the direct measurement error was used for species probed by fewer lines.

However, there is more than just the measurement error to consider. The chosen stellar model will also affect the derived abundances. The three important parameters in the model are: temperature, gravity and micro-turbulence velocity. Each of these influences the final abundance in a different way. We estimated the uncertainty in each of these three parameters using the corresponding statistical errors on the slopes and the (Fe I-Fe II) difference and computed the resultant change in abundance for all elemental ratios. Table 3 shows the abundance offset generated by each parameter, and the combined effect of all three (added quadratically).

A summary of our abundance analysis is available in Table A.2 (Fornax) and Table A.3 (M 15) where we present all of our elements with the associated error estimates and the number of lines used to compute the ratio. Only the $E W$ measurement

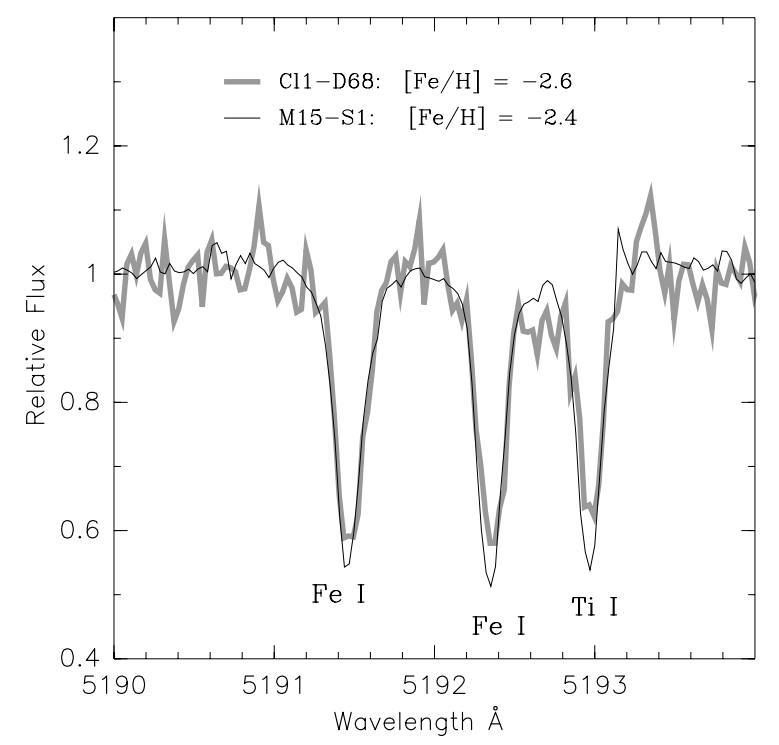

Fig. 5. The comparison between C11-D68 and M 15-S1. These are two RGB stars with similar stellar parameters but a difference in $[\mathrm{Fe} / \mathrm{H}]$ of 0.2 dex.

error is used in plots and tables, and Fe I is used to determine our $[\mathrm{el} / \mathrm{Fe}]$ ratios.

\section{Interpretation}

\subsection{The Iron abundance}

Table 4 compares our mean $[\mathrm{Fe} / \mathrm{H}]$ with the latest results of two different classical methods: RGB slope fitting and integrated spectroscopy. Our abundances appear 0.3 to 0.5 dex lower than previous estimates. Most of this discrepancy is attributable to different reference calibrators. Indeed, both the integrated spectroscopy and isochrone fitting are based on the Zinn \& West (1984) metallicity scale which places M 15 at $\langle[\mathrm{Fe} / \mathrm{H}]\rangle=-2.15$ and $\mathrm{M} 92$ at $\langle[\mathrm{Fe} / \mathrm{H}]\rangle=-2.24$. In contrast, high resolution spectroscopic analyses consistently find $\langle[\mathrm{Fe} / \mathrm{H}]\rangle=-2.4$ for M 15, including Sneden et al. (1997) and this present work. Meanwhile, $\mathrm{M} 92$ is found to be $\langle[\mathrm{Fe} / \mathrm{H}]\rangle=-2.34$ (Sneden et al. 2000). The difference between high resolution spectroscopy and the other indirect methods, due to differences in calibration, is therefore of the order of $0.25 \mathrm{dex}$, the rest of the discrepancy might be due to the propagation of errors, and indeed appears of the order of the quoted error bars $( \pm 0.2 \mathrm{dex})$. In conclusion, although the absolute value of metallicities presented in the works quoted in Table 4 do not appear accurate, the comparison made by the authors with M 15 and M 92, the most metal-poor clusters known in our Galaxy, is correct. Our analysis reveals that Cluster 1 , at $\langle[\mathrm{Fe} / \mathrm{H}]\rangle=-2.5$, is actually the most metal-poor globular cluster yet observed. It is clearly more metal-poor than M 15, with weaker iron lines, as can be seen in Fig. 5, where we compare C11-D68 and M 15-S1, two RGB stars of similar temperature, surface gravity and micro-turbulence velocity.

\subsection{The Alpha elements}

Alpha elements come predominantly from type II supernovae, unlike $\mathrm{Fe}$ which comes predominantly from type Ia SN (McWilliam 1997; Tinsley 1979). The $[\alpha / \mathrm{Fe}]$ ratios frequently display different patterns with respect to Fe in different environments (e.g., Shetrone et al. 2001). They are typically overabundant by +0.3 to +0.4 dex in Galactic globular cluster stars and 
Table 4. Recent metallicity estimates from different methods.

\begin{tabular}{rrrrr}
\hline \hline Cluster 1 & Cluster 2 & Cluster 3 & Method & Reference \\
\hline$-2.5 \pm 0.1$ & $-2.1 \pm 0.1$ & $-2.4 \pm 0.1$ & Individual stars, HR spectra & This work \\
N/A & $-1.76 \pm 0.41$ & $-1.84 \pm 0.18$ & Integrated light spectra & Strader et al. (2003) \\
$-2.20 \pm 0.20$ & $-1.78 \pm 0.20$ & $-1.96 \pm 0.20$ & RGB Slope & Buonanno et al. (1998) \\
\hline
\end{tabular}

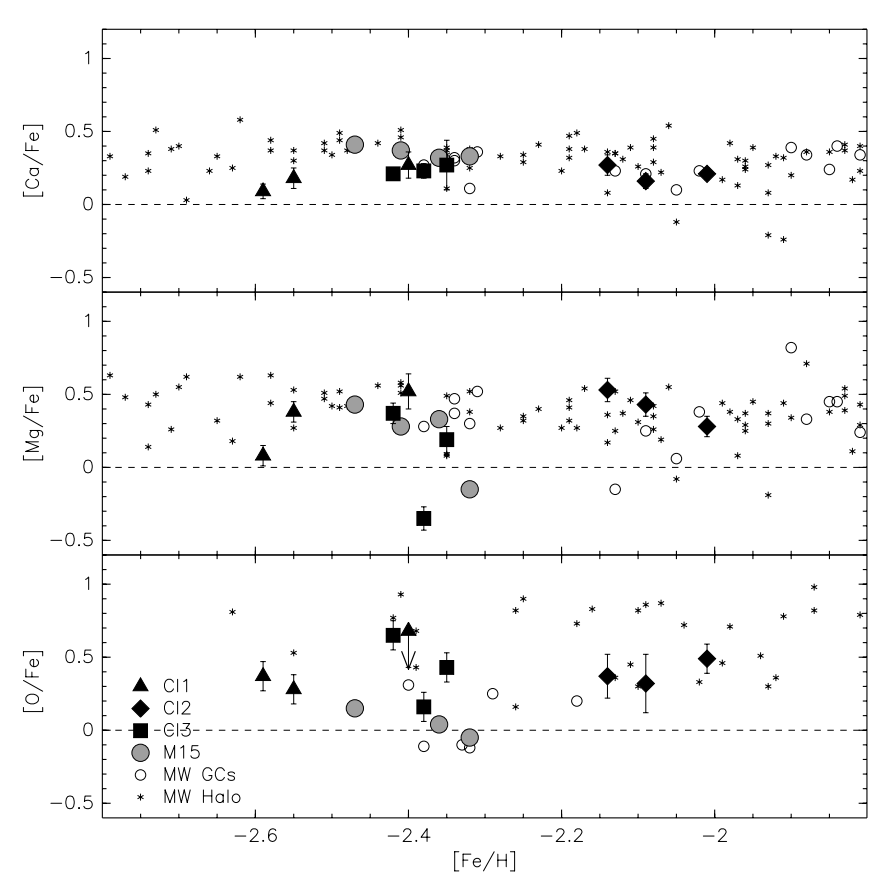

Fig. 6. Alpha elements abundances as a function of $[\mathrm{Fe} / \mathrm{H}]$. Filled triangles are for Cluster 1, filled diamonds are for Cluster 2 and filled circles are for Cluster 3. Asterisk are for our M 15 stars. Small grey dots are galactic stars and small empty circles are galactic GCs. Upper limits, when present, are shown with one sided arrows, replacing the error bars. See text for more details.

halo stars with respect to solar, as expected in old components where only SNe II have had time to contribute to the chemical enrichment.

In Fig. 6, we plot the abundance ratios for $\alpha$-elements $\mathrm{Ca}, \mathrm{Mg}$ and $\mathrm{O}$ in Fornax dSph globular clusters 1, 2 and 3. Also plotted are the four M 15 control stars and, as smaller symbols, Galactic halo stars, taken from the compilation of Venn et al. (2004) and Galactic globular cluster stars from the compilation (averaged by cluster) of Pritzl et al. (2005), except for [O/Fe] points, which are from Shetrone et al. (1996a, individual stars, not averages.) The abundances of $\mathrm{Ca}, \mathrm{Mg}$ and $\mathrm{O}$ are all above the solar value (just like the Galactic halo and globular cluster stars) with a small dispersion and small error bars. There are a couple of Fornax dSph globular cluster stars with clearly anomalous $\mathrm{O}$ and $\mathrm{Mg}$ abundance, and they will be discussed later in Sect. 4.3. The Fornax dSph globular cluster $\alpha$-element ratios appear to follow the same patterns found in Galactic globular cluster stars, suggesting that the oldest epoch of globular cluster formation is very similar in these two different environments. The overabundance of $\alpha$-elements seen in Galactic globular clusters stars may be interpreted as the number of massive stars present in the early history of our Galaxy assuming that the main contributor to $\alpha$-elements is $\mathrm{SNe}$ II explosions from massive stars. The same over abundance is seen in Fornax dSph globular clusters so this enrichment pattern is not only present in our Galaxy.

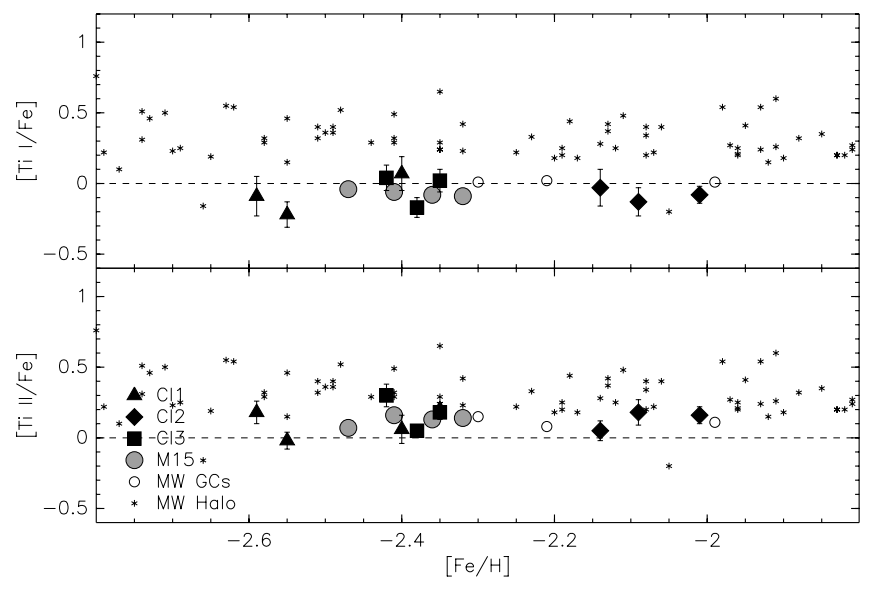

Fig. 7. Titanium abundances as a function of $[\mathrm{Fe} / \mathrm{H}]$. The symbols are the same as in Fig. 6. Separated Ti I and Ti II were not available for our halo stars, so a global $[\mathrm{Ti} / \mathrm{Fe}]$ is used for these points.

Titanium is shown in Fig. 7, where we chose to compare our results with the Galactic globular clusters studied by Shetrone et al. (2003) rather than the compilation of Pritzl et al. (2005) for homogeneity purposes. At first glance, Ti seems to be underabundant in the Fornax clusters with respect to halo stars in the Milky Way. However, they fall right on top of our M 15 and Shetrone's M 30, M 68 and M 55, close to a solar Ti/Fe ratio. However, we would like to stress that $[\mathrm{Ti} / \mathrm{Fe}]$ ratios of different authors can be on different scales (depending on the set of Ti lines used and the adopted $\log g f s)$, as well illustrated by M 15: the [Ti/Fe] ratios in M 15 found by Sneden et al. (1997, included in the Pritzl compilation) are $\sim 0.4$ dex higher than in our own analysis of M 15, but using Sneden's Ti lines, $\log g f s$ and $E W \mathrm{~s}$ (in the stars we have in common), our analysis yields the same value as Sneden's. We also notice a small systematic difference between the ratio of Ti I and Ti II over iron $(\sim 0.2 \mathrm{dex})$, that could be caused by $\log g f s$ (that could be on different scales for Ti I and Ti II) and/or non-LTE effects. We therefore conclude that, based on the comparison of our Fornax globular clusters with a fully compatible analysis of galactic globular clusters (our analysis of M 15 and three other clusters by Shetrone et al. 2003), there is no difference in the $\mathrm{Ti} / \mathrm{Fe}$ ratios observed in Fornax and MW globular clusters.

\subsection{Deep mixing pattern}

Deep-mixing occurs when material processed deep inside a star finds its way to the upper atmosphere, thus modifying the original abundance pattern. Proton-capture nucleosynthesis converts $\mathrm{O}, \mathrm{N}, \mathrm{Ne}$ to $\mathrm{Na}$, and $\mathrm{Mg}$ to $\mathrm{Al}$ in the $\mathrm{H}$ fusion layer of evolved RGB stars. This means that a significant atmospheric depletion of $\mathrm{O}$ caused by deep-mixing should be accompanied by an enhancement of Na (Langer et al. 1993) and similarly an enhancement in Al should cause observable Mg depletion (Langer \& Hoffman 1995). Such patterns (anti-correlations of $\mathrm{O}-\mathrm{Na}$ and $\mathrm{Mg}-\mathrm{Al}$ ) are found in galactic globular cluster stars 


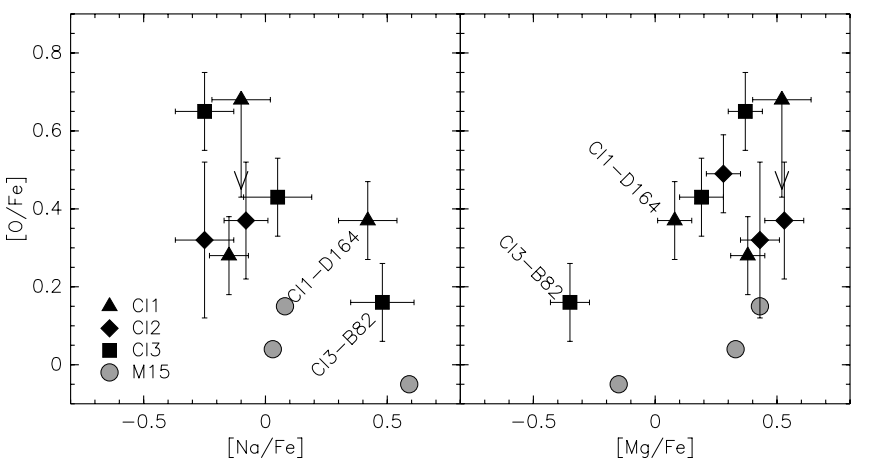

Fig. 8. Here we show the "Deep-Mixing" abundance anomaly. An anticorrelation of $\mathrm{O}-\mathrm{Na}$ on the left and a correlation of $\mathrm{O}-\mathrm{Mg}$ on the right. The symbols are the same as in Fig. 6.

but not in comparable field stars of our Galaxy (Gratton et al. 2004), or any other (e.g., Shetrone et al. 2001). It is assumed that this is caused by environmental effects within a star cluster but whether it is the result of deep-mixing within the RGB stars that are observed or the fossil traces of self-pollution of the globular cluster during its formation process, or a combination of the two, is not well understood.

Figure 8 shows that deep mixing patterns are not only found in galactic globular clusters and the old clusters of the Large Magellanic Cloud [LMC] (Hill et al. 2000) but also in clusters of much smaller dwarf spheroidal galaxies like Fornax. The anticorrelation $\mathrm{O}-\mathrm{Na}$ is visible in two $(\mathrm{Cl} 3-\mathrm{B} 82$ and $\mathrm{Cl1}-\mathrm{D} 164)$ of the nine stars we observed in the Fornax globular clusters displaying high $\mathrm{Na}$ and low $\mathrm{O}$ abundances (left panel), accompanied by low $\mathrm{Mg}$ abundances (correlation $\mathrm{O}-\mathrm{Mg}$, right panel). We cannot check directly whether the $\mathrm{Mg}$-Al anti-correlation also exists in these clusters, since we did not detect $\mathrm{Al}$ in our Fornax $\mathrm{dSph}$ globular cluster spectra, because the Al lines present in our spectral range are too weak. Our detection limit is about $14 \mathrm{~m} \AA$, which translates into an upper limit to $[\mathrm{Al} / \mathrm{Fe}]$ of 1.4 . Shetrone et al. (1996b) found that the usual enhancement of $\mathrm{Al}$ ranges from 0.5 to 1.0 dex, thus largely consistent with our upper limit.

\subsection{Iron-peak elements}

The Fe-peak elements we observed in the Fornax dSph globular clusters are $\mathrm{Cr}, \mathrm{Ni}$ and $\mathrm{Zn}$, and they are shown in Fig. 9. Comparison points for Galactic globular clusters are from the compilation of Pritzl et al. (2005), and Galactic halo stars are from the Hamburg-ESO (HERES) survey (Barklem et al. 2005) for Cr (top panel), from the compilation of Venn et al. (2004) for Ni (middle panel), and from Sneden et al. (1991) and Barklem et al. (2005) for Zn (lower panel).

$\mathrm{Cr}$ is believed to be produced mainly by incomplete explosive silicon burning (Woosley \& Weaver 1995). Despite large error bars in our measurements of the Fornax dSph globular cluster stars, there seems to be an increase (by $\sim 0.3 \mathrm{dex}$ ) of the $[\mathrm{Cr} / \mathrm{Fe}]$ ratio between the two more metal-poor clusters and the more metal-rich Cluster 2. Such a trend of increasing $[\mathrm{Cr} / \mathrm{Fe}]$ with increasing $[\mathrm{Fe} / \mathrm{H}]$ has been observed in Galactic field stars (McWilliam et al. 1995; Carretta et al. 2002), leading to a similar $\sim 0.3$ dex increase, but over a much wider metallicity range $(-3.5$ to -2.). Newer, high quality observations by Cayrel et al. (2004) of Galactic halo stars further reduced the observed slope of increasing $[\mathrm{Cr} / \mathrm{Fe}]$ with increasing metallicity to $\sim 0.15$ dex over a $[\mathrm{Fe} / \mathrm{H}]$ range from -2.5 to -4 dex, with an extremely small intrinsic scatter $(\sigma=0.05 \mathrm{dex})$. The higher $[\mathrm{Cr} / \mathrm{Fe}]$ abundance

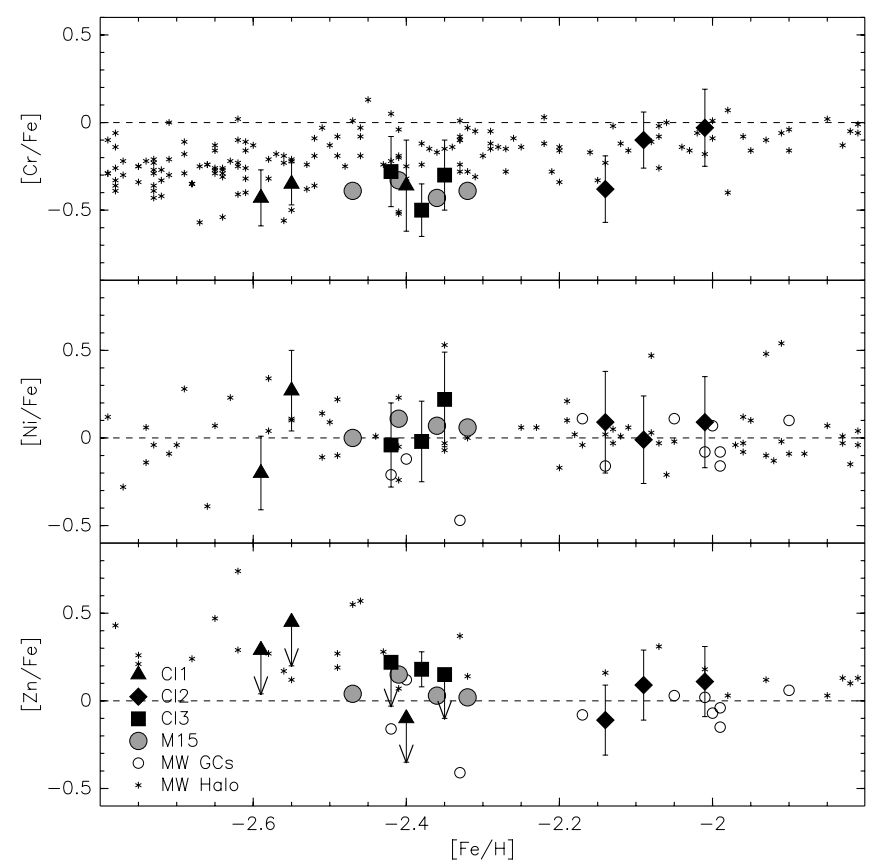

Fig. 9. Iron-peak elements abundances as a function of $[\mathrm{Fe} / \mathrm{H}]$. The symbols are the same as in Fig. 6.

observed in Cluster 2 therefore seems unlikely, and is probably caused by our observational errors.

$\mathrm{Ni}$ is believed to be produced in complete explosive silicon burning. We don't expect any relation between $[\mathrm{Ni} / \mathrm{Fe}]$ as a function of $[\mathrm{Fe} / \mathrm{H}]$, based on what we see in the MW. Even at this low metallicity, the relation is flat with a value close to zero, within the error bars, as we can see in Fig. 9. This is consistent with the majority of Galactic globular clusters, open clusters and halo stars (Sneden et al. 2004). So yet again, the Fornax dSph globular clusters are similar to the normal Galactic globular clusters.

$\mathrm{Zn}$ has the same origin as $\mathrm{Ni}$, but it has been suggested (Heger \& Woosley 2002) that it could also be formed by neutron capture, and be either an $r$-process or an $s$-process element. Our results, more than half of which are upper limits, are consistent with Galactic values.

\subsection{Heavy elements}

The heavy elements Y, Ba and Eu in the Fornax dSph globular clusters are plotted in Fig. 10. [Y/Fe] appears to be consistent with what is observed in Galactic globular clusters. However, Cluster 1 and 3 (the two most metal-poor) appear to have higher $[\mathrm{Ba} / \mathrm{Fe}]$ than average for Galactic globular clusters. As shown in Fig. 4), europium is measured from a single weak line, and could only be detected in Cluster 3 (all other Fornax points in this plot are upper limits), in which $[\mathrm{Eu} / \mathrm{Fe}]$ is particularly high, above the typical range for Galactic globular clusters.

$\mathrm{Ba}$ and $\mathrm{Y}$ are neutron-capture elements which are, in the solar system, dominated by the $s$-process, a process due to low to intermediate-mass Asymptotic Giant Branch (AGB) stars, with only a minor contribution from the $r$-process. Eu on the other hand is almost entirely dominated by the $r$-process, which requires more extreme neutron fluxes, such as SN II explosions (McWilliam 1997) associated with massive stars. In the Milky Way, with decreasing metallicities the $s$-process contribution gradually decreases (consistent with the timescale of AGB evolution) so that below $\sim-2.5 \mathrm{dex}$, both in field and 


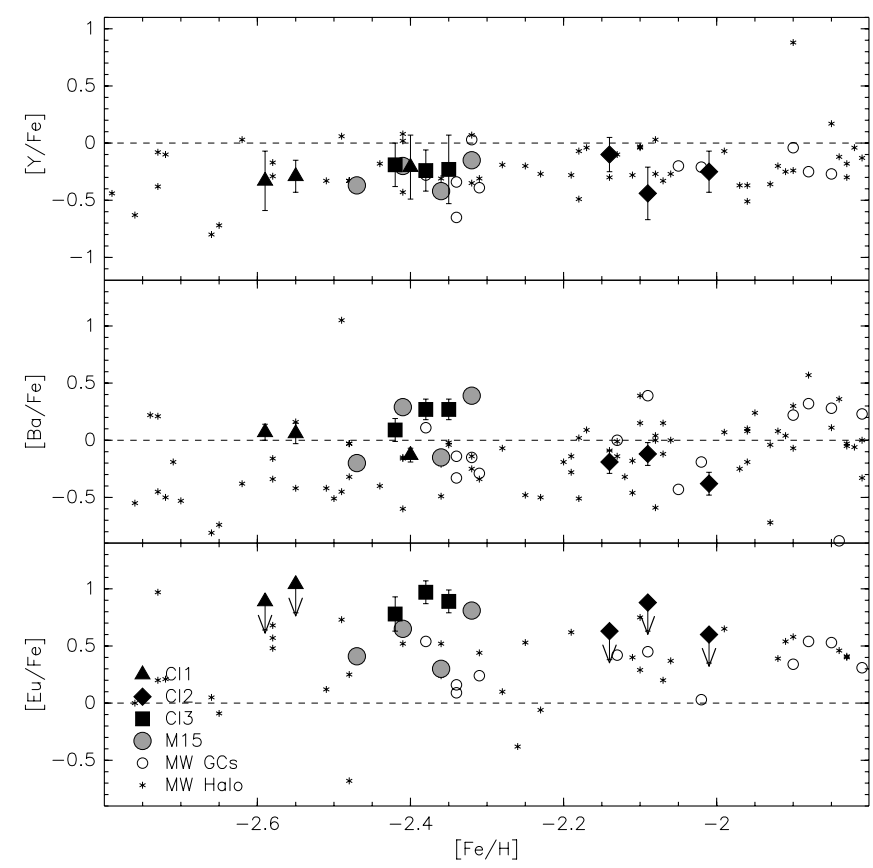

Fig. 10. Heavy elements abundances as a function of $[\mathrm{Fe} / \mathrm{H}]$. The symbols are the same as in Fig. 6.

globular clusters stars, all heavy elements are dominated exclusively by the $r$-process. (Johnson et al. 2001; James et al. 2004; Barklem et al. 2005). In Cluster 3, we detect, not only Eu, but also other heavy elements represented by weak lines preventing detection in the other clusters: $\mathrm{Nd}$ and La. In Fig. 11, we compare Cluster $3 \log (\epsilon)^{3}$ values to the solar system $r$ and $s$-process abundances (Burris et al. 2000). The solar system elemental abundances have been shifted by the difference between the mean values of Eu for Cluster 3 and the solar system abundance distribution ( $-1.55 \mathrm{dex})$. Clearly, the abundances of most elements in the Fornax globular clusters match the solar system $r$-process pattern within the observational uncertainties (with the exception of La which seems to be matched by neither the $r$ - nor the $s$-process patterns). Cluster 3 stars are obviously very close to the $r$-process expectations, confirming that, similarly to the most metal-poor globular clusters in the galactic Halo (M 15, M 92, M 68), cluster 3 is also dominated by the $r$-process. This is also confirmed by the $[\mathrm{Ba} / \mathrm{Eu}]$ ratio observed in the three Cluster 3 stars $[\mathrm{Ba} / \mathrm{Eu}]=-0.62,-0.69,-0.7$ $( \pm 0.20)$, very close to the -0.69 for the $r$-process component in the solar system (as compared to +1.15 for the $s$-process, Arlandini et al. 1999). The upper limits for Eu in Clusters 1 and 2, although not decisive, are also compatible with a pure $r$-process enrichment $([\mathrm{Ba} / \mathrm{Eu}]>-1.00$ to -0.82$)$. This result indicates that, in Fornax dSph as in our Galaxy, heavier neutron capture elements in the lowest metallicity stars have only very weak $s$-process contribution. Or in other words, that heavy elements in Fornax dSph globular clusters, as in M 15, are formed principally through the $r$-process.

The high neutron-capture element content of Cluster 3, that we attribute to the $r$-process, is similar for all three stars, and above the upper edge of the range of values traditionally covered by the Galactic globular clusters (Pritzl et al. 2005). $R$-process enrichments of this order or even higher are found in Galactic halo field stars (Barklem et al. 2005), but as far as Galactic

${ }^{3}$ The scale used for $\log (\epsilon)$ is the standard astronomical scale $\left(\log _{10}\left(N_{\mathrm{el}} / N_{\mathrm{H}}\right)+12\right)$.

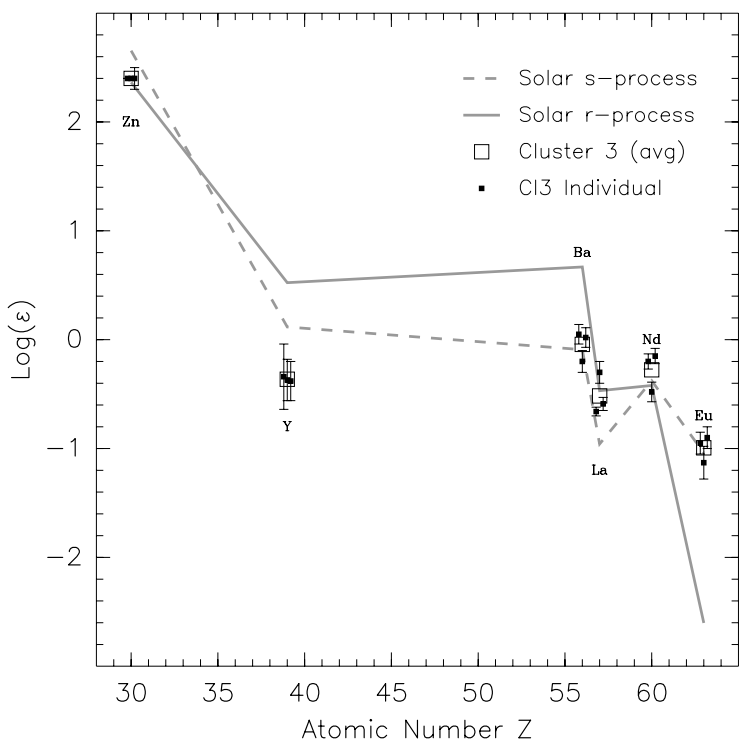

Fig. 11. The relative contributions of the $r$ - and $s$-processes for the heavy elements in Cluster 3 (filled circle). The solar $r$ - and $s$-process abundances, traced by a dotted and a full line respectively, are taken from Burris et al. (2000). They are shifted by the difference between Cluster 3 and the solar system abundance for $\mathrm{Eu}(-1.55 \mathrm{dex})$.

globular clusters are concerned, the only case known to date is M 15. Sneden et al. (1997, 2000) have established that M 15 has a stellar bi-modality with one group being strongly overabundant in $[\mathrm{Eu} / \mathrm{Fe}]$ (and $[\mathrm{Ba} / \mathrm{Fe}]$ ) compared to the other. Our observations of three stars in Cluster 3 do not provide sufficient statistics to determine if this cluster also has a bimodality (with our 3 stars by chance happening to belong to the high Eu group) or if all stars in Cluster 3 are Eu-rich.

Finally, despite the dispersion in $\mathrm{Ba}$ that seems to exist among the three Fornax globular clusters (Cluster 3 being the most $\mathrm{Ba}$ and $\mathrm{Eu}$ rich), $\mathrm{Y}$ is very similar from cluster to cluster, and comparable to the Galactic abundances of this element (globular clusters and field stars). This also leads Cluster 3 to have $\mathrm{a} \mathrm{Ba} / \mathrm{Y}$ ratio higher than in the two other clusters $([\mathrm{Ba} / \mathrm{Y}]=$ +0.43 compared to +0.0 in Cluster 2 , and marginally higher than the +0.28 dex observed in Cluster 1$)$. Interestingly, the $[\mathrm{Ba} / \mathrm{Y}]$ observed in the three Fornax clusters are yet again very similar to that of the Galactic globular clusters and halo field stars, whereas the (on average more metal-rich) field stars in dwarf spheroidal galaxies have been shown to display systematically higher $[\mathrm{Ba} / \mathrm{Y}]$ than their galactic counterparts (Venn et al. 2004).

\section{Conclusions}

We have compared the properties of the globular clusters belonging to the Fornax dSph with those of the Milky Way with unprecedented accuracy. The Fornax dSph contains clusters with a range of properties such as metallicity, central concentration and Horizontal Branch structure. For the first time detailed chemical abundances have been derived for a sample of stars in a globular cluster system in an external galaxy, apart from the Magellanic Clouds. Despite their very different mass, morphology and global star formation history, the Fornax dSph and the Milky Way appear to have experienced the same very early enrichment conditions and in particular similar nucleosynthesis. This is summarised in Fig. 12, where the mean elemental abundances, each being weighted by its error, of the three Fornax globular clusters and M 15 are compared. The abundance 


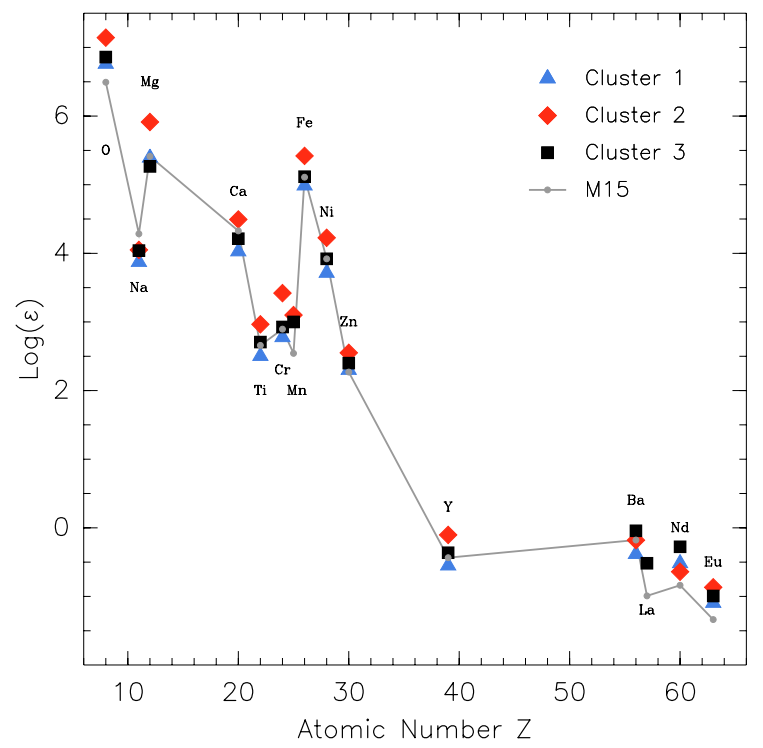

Fig. 12. The cluster mean elemental abundances of the three Fornax dSph globular clusters and M 15. Each individual stellar abundance has been weighted by its error. Cluster 1 is identified by a filled square, Cluster 2 by a star, Cluster 3 by a cross and M 15 by a triangle.

patterns of the individual stars in Milky Way globular clusters and Fornax globular clusters match each other almost perfectly. We find that the star-to-star abundance dispersion in the Fornax clusters is modest and compatible with similar observations of Galactic globular clusters.

We have definitively established that the Fornax globular Clusters 1, 2 and 3 are very metal-poor, slightly poorer than previous estimates, with respectively $\langle[\mathrm{Fe} / \mathrm{H}]\rangle=-2.5,-2.1$ and -2.4. Part of the discrepancy with previous studies is explained by the different reference calibrations used. Cluster 1 is now the most metal-poor globular cluster known, however the difference between Cluster 1 and M 92 or M 15 in the Milky Way is small. There seems to be universal lower limit to the metallicity at which star clusters form, which is higher than that of field stars in the halo our Galaxy, where significant numbers of stars are found with $[\mathrm{Fe} / \mathrm{H}]<-4$. It is also clear that, as in our Galaxy, the ratio of the number of globular cluster to the number of field stars strongly decreases with rising metallicity (Harris \& Harris 2002).

Clusters 1, 2 and 3 were clearly formed promptly and early in the history of Fornax dSph, alike the Milky Way globular clusters. They are over abundant in $\alpha$-elements $(\mathrm{O}, \mathrm{Mg}, \mathrm{Ca})$ at a similar level to Galactic clusters at identical $[\mathrm{Fe} / \mathrm{H}]$, and the heavy element abundances (Y, Ba, Eu) in the 3 clusters are compatible with dominant $r$-process enrichment. Finally, the Fe-peak elements are also very similar to Galactic globular cluster values, with $[\mathrm{Ni} / \mathrm{Fe}]$ being unambiguously solar in all three clusters and $\mathrm{Zn}$ and $\mathrm{Cr}$ are also compatible with Galactic values.

The analogy between Galactic and Fornax dSph holds even in the rare cases and anomalies: (i) Eu is extremely overabundant in Cluster 3 stars. The only Galactic counterpart known to date is $\mathrm{M} 15$; (ii) Cl1-D164 and Cl3-B82 show low $\mathrm{O}$ and $\mathrm{Mg}$ associated with a high $\mathrm{Na}$ abundance, thus establishing an $\mathrm{O}-\mathrm{Na}$ anticorrelation and $\mathrm{O}-\mathrm{Mg}$ correlation. This is the same deep-mixing pattern observed Galactic star clusters, and old LMC clusters.
The effort towards a comprehensive description of the formation and evolution of the Fornax dSph will soon benefit from the analysis of VLT/FLAMES high resolution spectra of a hundred field stars (Letarte et al., in preparation). It will then be possible to describe the chemical enrichment and nucleosynthetic processes dominant for the field star population compared to that found in the globular clusters, and to see when and if the similarities in enrichment patterns with our Galaxy end.

Acknowledgements. We gratefully acknowledge Carlo Emanuele Corsi and Roberto Buonanno for providing their photometry for our target selection. E.T. gratefully acknowledges support from a fellowship of the Royal Netherlands Academy of Arts and Sciences. B.L. is funded by a grant from the Netherlands Organisation for Scientific Research (NWO). P.J. and G.M. gratefully acknowledge support from the Swiss National Science Foundation (SNSF).

\section{References}

Alonso, A., Arribas, S., \& Martínez-Roger, C. 2001, A\&A, 376, 1039 Arlandini, C., Käppeler, F., Wisshak, K., et al. 1999, ApJ, 525, 886 Barklem, P. S., Christlieb, N., Beers, T. C., et al. 2005, A\&A, 439, 129 Buonanno, R., Corsi, C. E., Castellani, M., et al. 1999, AJ, 118, 1671 Buonanno, R., Corsi, C. E., Fusi Pecci, F., Hardy, E., \& Zinn, R. 1985, A\&A, 152,65

Buonanno, R., Corsi, C. E., Zinn, R., et al. 1998, ApJ, 501, L33

Burris, D. L., Pilachowski, C. A., Armandroff, T. E., et al. 2000, ApJ, 544, 302

Carretta, E., Gratton, R., Cohen, J. G., Beers, T. C., \& Christlieb, N. 2002, AJ, 124,481

Cayrel, R., Depagne, E., Spite, M., et al. 2004, A\&A, 416, 1117

Dekker, H., D’Odorico, S., Kaufer, A., Delabre, B., \& Kotzlowski, H. 2000, in Optical and IR Telescope Instrumentation and Detectors, ed. M. Iye, \& A. F. Moorwood, Proc. SPIE, 4008, 534

Demers, S., Grondin, L., \& Kunkel, W. E. 1990, PASP, 102, 632

Gratton, R., Sneden, C., \& Carretta, E. 2004, ARA\&A, 42, 385

Harris, W. E., \& Harris, G. L. H. 2002, AJ, 123, 3108

Heger, A., \& Woosley, S. E. 2002, ApJ, 567, 532

Hill, V., François, P., Spite, M., Primas, F., \& Spite, F. 2000, A\&A, 364, L19

Hodge, P. W. 1961, PASP, 73, 328

James, G., François, P., Bonifacio, P., et al. 2004, A\&A, 427, 825

Johnson, J. A., \& Bolte, M. 2001, ApJ, 554, 888

Jorgensen, U. G., \& Jimenez, R. 1997, A\&A, 317, 54

Kustner, F. 1921, Veroeffentlichungen des Astronomisches Institute der Universitaet Bonn, 15, 1

Langer, G. E., Hoffman, R., \& Sneden, C. 1993, PASP, 105, 301

Langer, G. E., \& Hoffman, R. D. 1995, PASP, 107, 1177

Lotz, J. M., Miller, B. W., \& Ferguson, H. C. 2004, ApJ, 613, 262

McWilliam, A. 1997, ARA\&A, 35, 503

McWilliam, A., Preston, G. W., Sneden, C., \& Searle, L. 1995, AJ, 109, 2757

Pritzl, B. J., Venn, K. A., \& Irwin, M. 2005, AJ, 130, 2140

Sandage, A. 1970, ApJ, 162, 841

Saviane, I., Held, E. V., \& Bertelli, G. 2000, A\&A, 355, 56

Seth, A., Olsen, K., Miller, B., Lotz, J., \& Telford, R. 2004, AJ, 127, 798

Shapley, H. 1938, Nature, 142, 715

Shetrone, M., Venn, K. A., Tolstoy, E., et al. 2003, AJ, 125, 684

Shetrone, M. D. 1996a, AJ, 112, 1517

Shetrone, M. D. 1996b, AJ, 112, 2639

Shetrone, M. D., Côté, P., \& Sargent, W. L. W. 2001, ApJ, 548, 592

Sneden, C., Gratton, R. G., \& Crocker, D. A. 1991, A\&A, 246, 354

Sneden, C., Ivans, I. I., \& Fulbright, J. P. 2004, in Origin and Evolution of the Elements, 172

Sneden, C., Kraft, R. P., Shetrone, M. D., et al. 1997, AJ, 114, 1964

Sneden, C., Pilachowski, C. A., \& Kraft, R. P. 2000, AJ, 120, 1351

Spite, M. 1967, Ann. Astrophys., 30, 211

Stetson, P. B., Hesser, J. E., \& Smecker-Hane, T. A. 1998, PASP, 110, 533

Strader, J., Brodie, J. P., Forbes, D. A., Beasley, M. A., \& Huchra, J. P. 2003, AJ, 125,1291

Tinsley, B. M. 1979, ApJ, 229, 1046

van den Bergh, S. 2005 [arXiv:astro-ph/0509811]

Venn, K. A., Irwin, M., Shetrone, M. D., et al. 2004, AJ, 128, 1177

Woosley, S. E., \& Weaver, T. A. 1995, ApJS, 101, 181

Zinn, R., \& West, M. J. 1984, ApJS, 55, 45 
B. Letarte et al.: VLT/UVES spectroscopy in Fornax GCs, Online Material $p 1$

\section{Online Material}


B. Letarte et al.: VLT/UVES spectroscopy in Fornax GCs, Online Material p 2

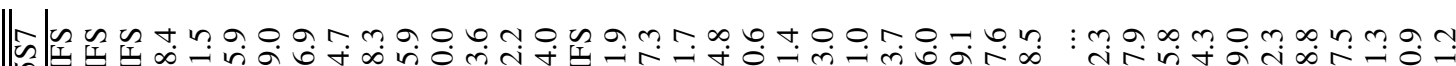

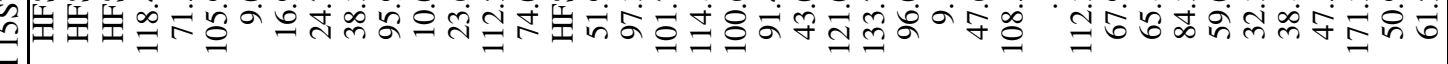

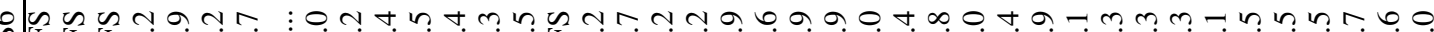

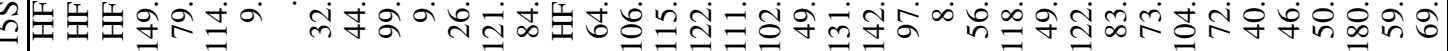

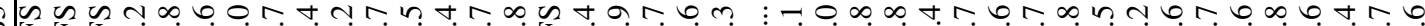
I

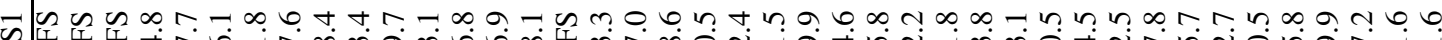

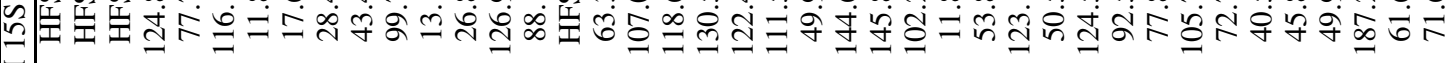

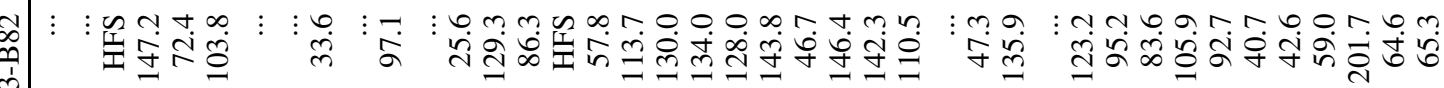

-

w : nono :

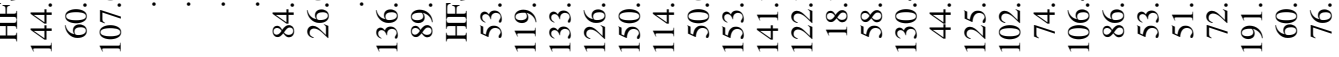

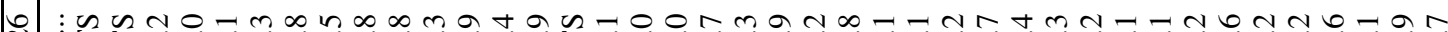

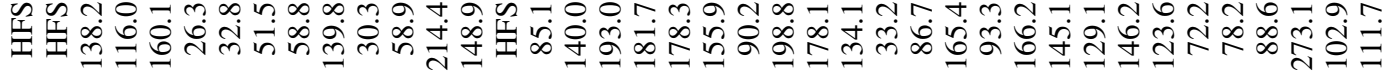

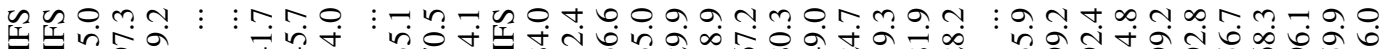

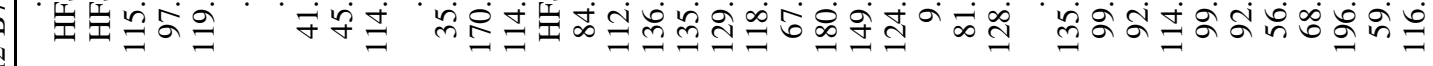

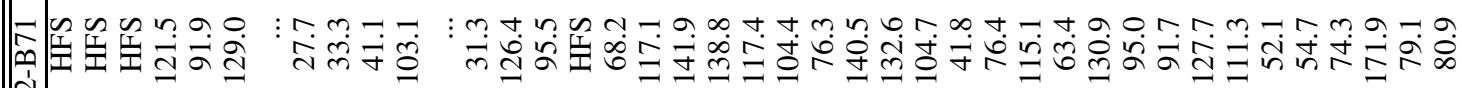

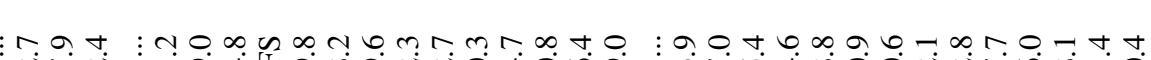

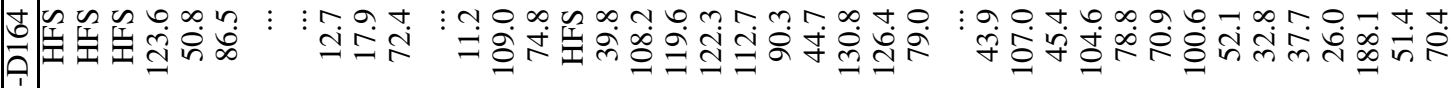

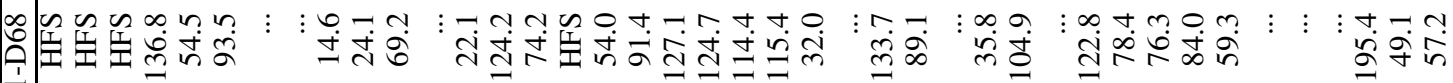

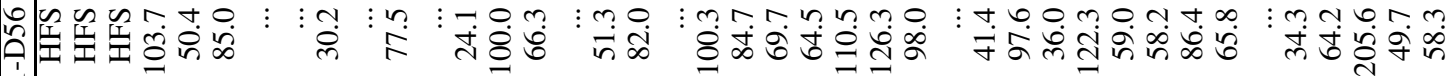

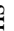

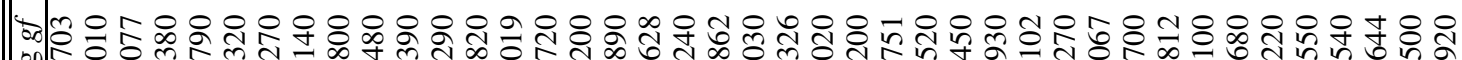

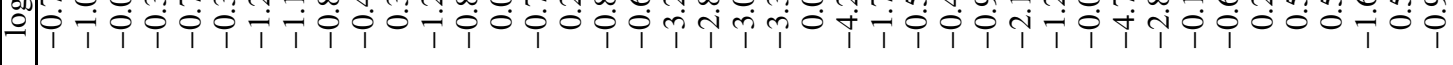

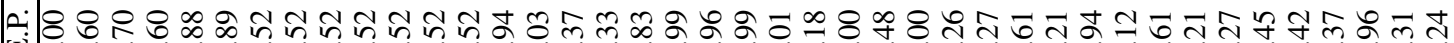
4000- 0 -

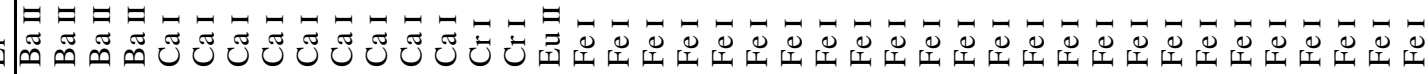

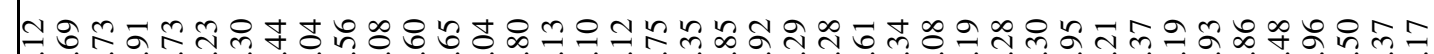

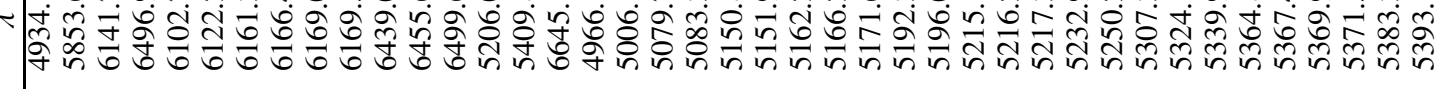




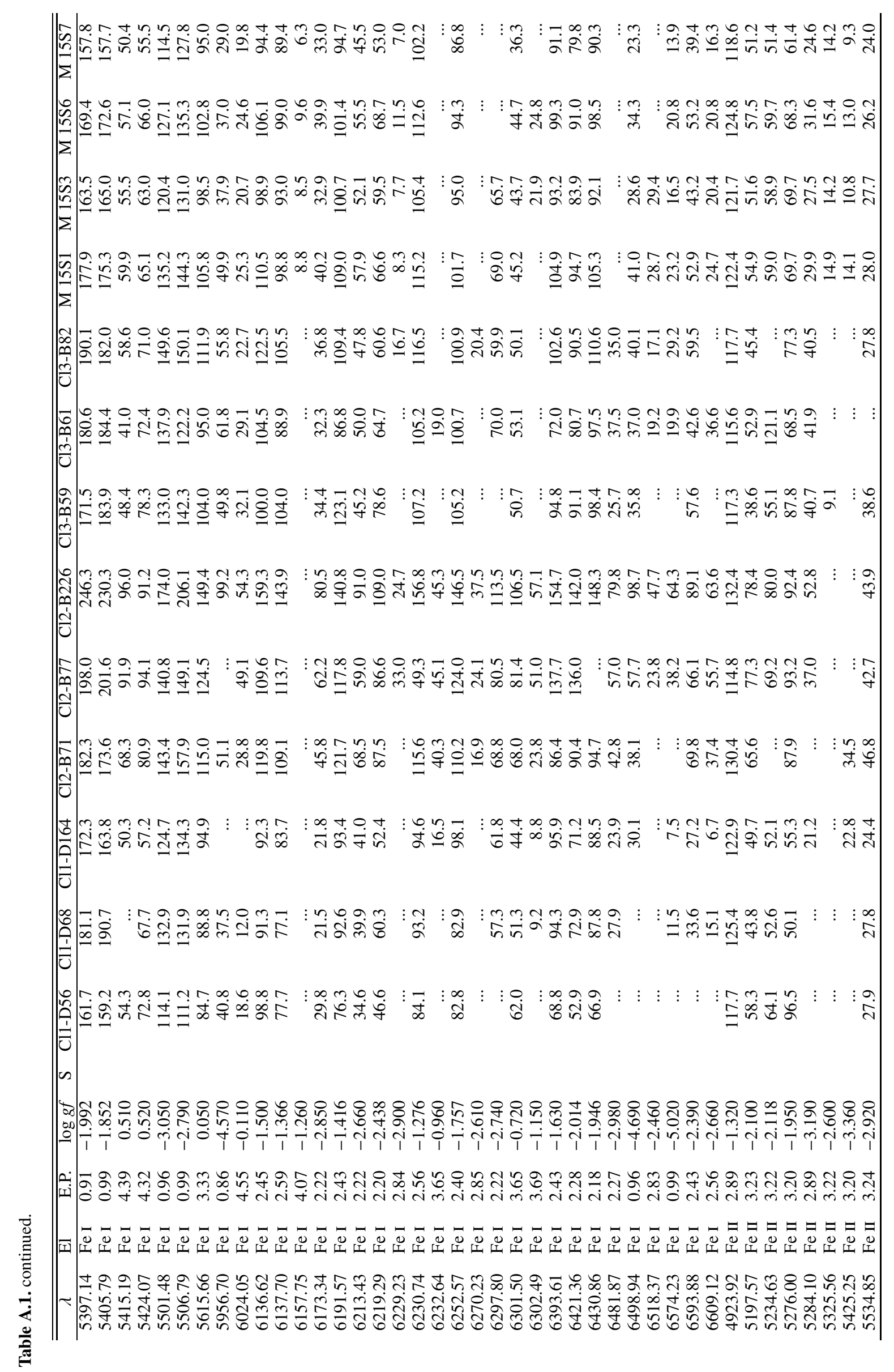




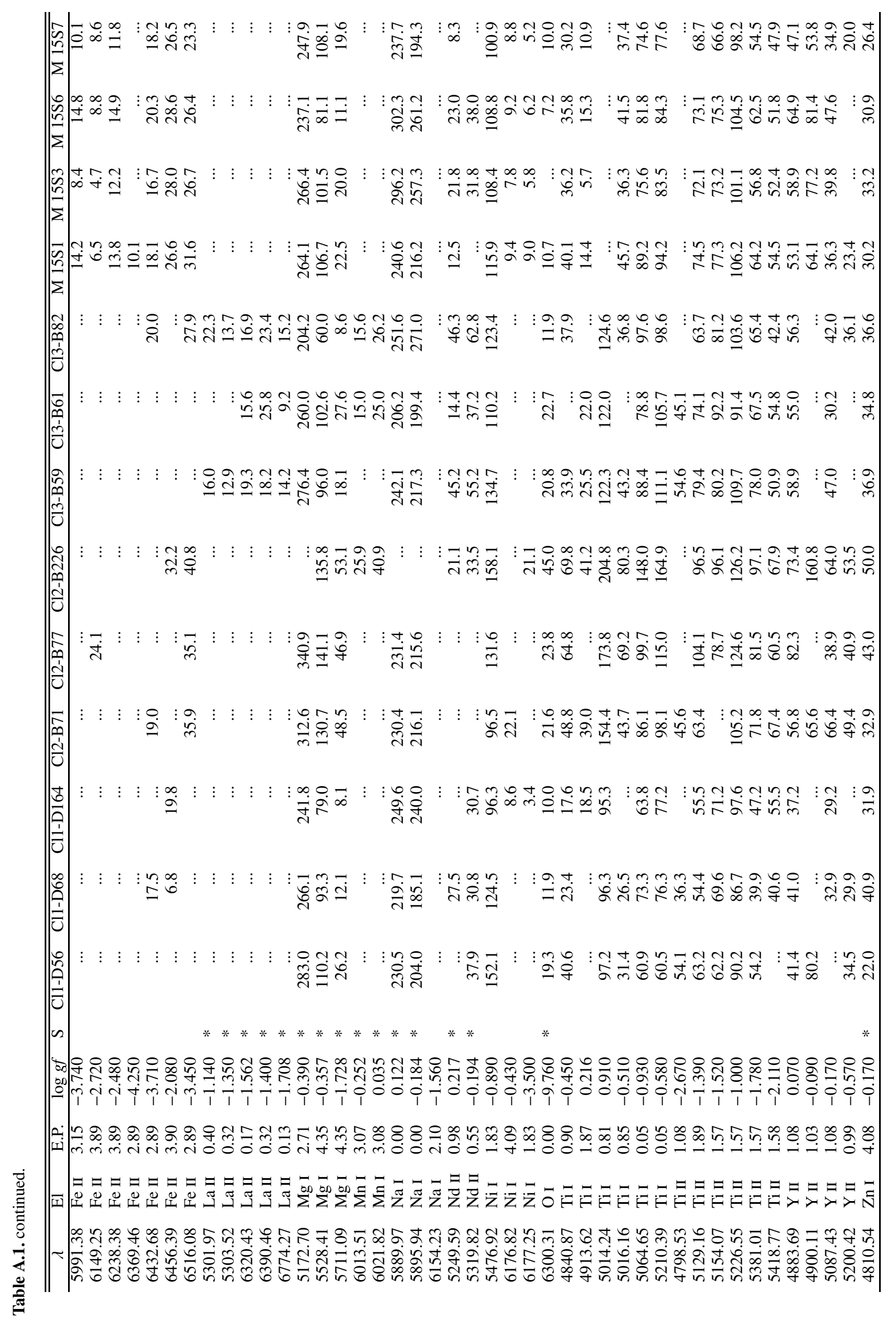


B. Letarte et al.: VLT/UVES spectroscopy in Fornax GCs, Online Material p 5

Table A.2. Fornax elemental ratios.

\begin{tabular}{|c|c|c|c|c|c|c|c|c|c|}
\hline & Cl1-D56 & $\sigma$ & $N_{\text {lines }}$ & Cl1-D68 & $\sigma$ & $N_{\text {lines }}$ & Cl1-D164 & $\sigma$ & $\overline{N_{\text {lines }}}$ \\
\hline$[\mathrm{Ba} \mathrm{II} / \mathrm{Fe} \mathrm{I}]$ & -0.13 & 0.06 & 4 & 0.06 & 0.09 & 4 & 0.07 & 0.07 & 4 \\
\hline$[\mathrm{Ca} \mathrm{I} / \mathrm{Fe} \mathrm{I}]$ & 0.27 & 0.09 & 5 & 0.18 & 0.07 & 5 & 0.09 & 0.05 & 4 \\
\hline$[\mathrm{Cr} \mathrm{I} / \mathrm{Fe} \mathrm{I}]$ & -0.36 & 0.26 & 2 & -0.35 & 0.12 & 2 & -0.43 & 0.16 & 2 \\
\hline$[\mathrm{Eu} \mathrm{II} / \mathrm{Fe} \mathrm{I}]$ & & & 0 & $<1.04$ & 0.00 & 1 & $<0.89$ & 0.00 & 1 \\
\hline$[\mathrm{Fe} \mathrm{I} / \mathrm{H}]$ & -2.40 & 0.03 & 40 & -2.55 & 0.03 & 39 & -2.59 & 0.03 & 45 \\
\hline$[\mathrm{Fe} \mathrm{II} / \mathrm{H}]$ & -2.43 & 0.06 & 4 & -2.62 & 0.11 & 5 & -2.56 & 0.06 & 7 \\
\hline$[\mathrm{La} \mathrm{II} / \mathrm{Fe} \mathrm{I}]$ & & & 0 & & & 0 & & & 0 \\
\hline$[\mathrm{Mg} \mathrm{I} / \mathrm{Fe} \mathrm{I}]$ & 0.52 & 0.12 & 3 & 0.38 & 0.07 & 3 & 0.08 & 0.07 & 3 \\
\hline$[\mathrm{Mn} \mathrm{I} / \mathrm{Fe} \mathrm{I}]$ & & & 0 & & & 0 & & & 0 \\
\hline$[\mathrm{Na} / / \mathrm{Fe} \mathrm{I}]$ & -0.10 & 0.12 & 2 & -0.15 & 0.08 & 2 & 0.42 & 0.12 & 2 \\
\hline$[\mathrm{Nd} \mathrm{II} / \mathrm{Fe} \mathrm{I}]$ & 0.60 & 0.20 & 1 & 0.40 & 0.07 & 2 & 0.49 & 0.10 & 1 \\
\hline$[\mathrm{Ni} \mathrm{I} / \mathrm{Fe} \mathrm{I}]$ & $\ldots$ & & 0 & 0.27 & 0.23 & 1 & -0.20 & 0.21 & 1 \\
\hline$[\mathrm{O} \mathrm{I} / \mathrm{Fe} \mathrm{I}]$ & $<0.68$ & 0.00 & 1 & 0.28 & 0.10 & 1 & 0.37 & 0.10 & 1 \\
\hline$[\mathrm{Ti} \mathrm{I} / \mathrm{Fe} \mathrm{I}]$ & 0.07 & 0.12 & 5 & -0.22 & 0.09 & 5 & -0.09 & 0.14 & 5 \\
\hline [Ti II/Fe I] & 0.06 & 0.10 & 5 & -0.02 & 0.06 & 6 & 0.18 & 0.08 & 5 \\
\hline [Y II/Fe I] & -0.21 & 0.28 & 2 & -0.29 & 0.14 & 3 & -0.33 & 0.26 & 2 \\
\hline \multirow[t]{2}{*}[\mathrm{Zn}\mathrm{I}/\mathrm{Fe}\mathrm{I}]{} & $<-0.10$ & 0.00 & 1 & $<0.45$ & 0.00 & 1 & $<0.29$ & 0.00 & 1 \\
\hline & Cl2-B71 & $\sigma$ & $N_{\text {lines }}$ & Cl2-B77 & $\sigma$ & $N_{\text {lines }}$ & $\mathrm{Cl2}-\mathrm{B} 226$ & $\sigma$ & $N_{\text {lines }}$ \\
\hline$[\mathrm{Ba} \mathrm{II} / \mathrm{Fe} \mathrm{I}]$ & -0.19 & 0.10 & 4 & -0.12 & 0.10 & 3 & -0.38 & 0.10 & 3 \\
\hline$[\mathrm{Ca} \mathrm{I} / \mathrm{Fe} \mathrm{I}]$ & 0.27 & 0.07 & 7 & 0.16 & 0.05 & 6 & 0.21 & 0.04 & 9 \\
\hline$[\mathrm{Cr} \mathrm{I} / \mathrm{Fe} \mathrm{I}]$ & -0.38 & 0.19 & 2 & -0.10 & 0.16 & 2 & -0.03 & 0.22 & 2 \\
\hline$[\mathrm{Eu} \mathrm{II} / \mathrm{Fe} \mathrm{I}]$ & $<0.63$ & 0.00 & 1 & $<0.88$ & 0.00 & 1 & $<0.60$ & 0.00 & 1 \\
\hline$[\mathrm{Fe} \mathrm{I} / \mathrm{H}]$ & -2.14 & 0.03 & 50 & -2.09 & 0.04 & 47 & -2.01 & 0.02 & 47 \\
\hline$[\mathrm{Fe} \mathrm{II} / \mathrm{H}]$ & -2.06 & 0.06 & 6 & -2.03 & 0.07 & 6 & -2.01 & 0.04 & 8 \\
\hline$[\mathrm{La} \mathrm{II} / \mathrm{Fe} \mathrm{I}]$ & & & 0 & & & 0 & & & 0 \\
\hline$[\mathrm{Mg} \mathrm{I} / \mathrm{Fe} \mathrm{I}]$ & 0.53 & 0.08 & 3 & 0.43 & 0.08 & 3 & 0.28 & 0.07 & 2 \\
\hline$[\mathrm{Mn} \mathrm{I} / \mathrm{Fe} \mathrm{I}]$ & & & 0 & & & 0 & -0.28 & 0.07 & 2 \\
\hline$[\mathrm{Na} \mathrm{I} / \mathrm{Fe} \mathrm{I}]$ & -0.08 & 0.09 & 2 & -0.25 & 0.12 & 2 & & & 0 \\
\hline$[\mathrm{Nd} \mathrm{II} / \mathrm{Fe} \mathrm{I}]$ & $\ldots$ & $\ldots$ & 0 & $\ldots$ & $\ldots$ & 0 & -0.13 & 0.12 & 2 \\
\hline$[\mathrm{Ni} \mathrm{I} / \mathrm{Fe} \mathrm{I}]$ & 0.09 & 0.29 & 2 & -0.01 & 0.25 & 1 & 0.09 & 0.26 & 2 \\
\hline$[\mathrm{O} \mathrm{I} / \mathrm{Fe} \mathrm{I}]$ & 0.37 & 0.15 & 1 & 0.32 & 0.20 & 1 & 0.49 & 0.10 & 1 \\
\hline$[\mathrm{Ti} \mathrm{I} / \mathrm{Fe} \mathrm{I}]$ & -0.03 & 0.13 & 5 & -0.13 & 0.10 & 4 & -0.08 & 0.06 & 5 \\
\hline [Ti II/Fe I] & 0.05 & 0.07 & 5 & 0.18 & 0.09 & 4 & 0.16 & 0.06 & 5 \\
\hline$[\mathrm{Y} \mathrm{II} / \mathrm{Fe} \mathrm{I}]$ & -0.10 & 0.15 & 4 & -0.44 & 0.23 & 2 & -0.25 & 0.18 & 3 \\
\hline \multirow[t]{2}{*}[\mathrm{Zn}\mathrm{I}/\mathrm{Fe}\mathrm{I}]{} & -0.11 & 0.20 & 1 & 0.09 & 0.20 & 1 & 0.11 & 0.20 & 1 \\
\hline & Cl3-B59 & $\sigma$ & $N_{\text {lines }}$ & C13-B61 & $\sigma$ & $N_{\text {lines }}$ & C13-B82 & $\sigma$ & $N_{\text {lines }}$ \\
\hline$[\mathrm{Ba} \mathrm{II} / \mathrm{Fe} \mathrm{I}]$ & 0.27 & 0.09 & 3 & 0.09 & 0.10 & 3 & 0.27 & 0.09 & 2 \\
\hline$[\mathrm{Ca} \mathrm{I} / \mathrm{Fe} \mathrm{I}]$ & 0.27 & 0.17 & 4 & 0.21 & 0.03 & 6 & 0.23 & 0.05 & 5 \\
\hline$[\mathrm{Cr} \mathrm{I} / \mathrm{Fe} \mathrm{I}]$ & -0.30 & 0.20 & 2 & -0.28 & 0.20 & 2 & -0.50 & 0.15 & 2 \\
\hline$[\mathrm{Eu} \mathrm{II} / \mathrm{Fe} \mathrm{I}]$ & 0.89 & 0.10 & 1 & 0.78 & 0.15 & 1 & 0.97 & 0.10 & 1 \\
\hline$[\mathrm{Fe} \mathrm{I} / \mathrm{H}]$ & -2.35 & 0.02 & 44 & -2.42 & 0.03 & 50 & -2.38 & 0.03 & 48 \\
\hline$[\mathrm{Fe} \mathrm{II} / \mathrm{H}]$ & -2.30 & 0.09 & 5 & -2.31 & 0.09 & 4 & -2.36 & 0.06 & 8 \\
\hline$[\mathrm{La} \mathrm{II} / \mathrm{Fe} \mathrm{I}]$ & $<0.52$ & 0.04 & 5 & 0.95 & 0.10 & 1 & 0.62 & 0.06 & 3 \\
\hline$[\mathrm{Mg} \mathrm{I} / \mathrm{Fe} \mathrm{I}]$ & 0.19 & 0.09 & 3 & 0.37 & 0.07 & 3 & -0.35 & 0.08 & 3 \\
\hline $\mathrm{I} / \mathrm{Fe} \mathrm{I}]$ & & & 0 & 0.03 & 0.07 & 2 & -0.01 & 0.07 & 2 \\
\hline$[\mathrm{Na} \mathrm{I} / \mathrm{Fe} \mathrm{I}]$ & 0.05 & 0.14 & 2 & -0.25 & 0.12 & 2 & 0.48 & 0.13 & 2 \\
\hline$[\mathrm{Nd} \mathrm{II} / \mathrm{Fe} \mathrm{I}]$ & 0.65 & 0.07 & 2 & 0.44 & 0.09 & 2 & 0.73 & 0.07 & 2 \\
\hline$[\mathrm{Ni} \mathrm{I} / \mathrm{Fe} \mathrm{I}]$ & 0.22 & 0.27 & 1 & -0.04 & 0.24 & 1 & -0.02 & 0.23 & 1 \\
\hline$[\mathrm{O} \mathrm{I} / \mathrm{Fe} \mathrm{I}]$ & 0.43 & 0.10 & 1 & 0.65 & 0.10 & 1 & 0.16 & 0.10 & 1 \\
\hline$[\mathrm{Ti} \mathrm{I} / \mathrm{Fe} \mathrm{I}]$ & 0.02 & 0.08 & 6 & 0.04 & 0.09 & 4 & -0.17 & 0.07 & 5 \\
\hline [Ti II/Fe I] & 0.18 & 0.05 & 6 & 0.30 & 0.08 & 6 & 0.05 & 0.03 & 5 \\
\hline$[\mathrm{Y} \mathrm{II} / \mathrm{Fe} \mathrm{I}]$ & -0.23 & 0.30 & 2 & -0.19 & 0.19 & 2 & -0.24 & 0.18 & 3 \\
\hline$[\mathrm{Zn} \mathrm{I} / \mathrm{Fe} \mathrm{I}]$ & $<0.15$ & 0.00 & 1 & $<0.22$ & 0.00 & 1 & 0.18 & 0.10 & 1 \\
\hline
\end{tabular}


B. Letarte et al.: VLT/UVES spectroscopy in Fornax GCs, Online Material p 6

Table A.3. M 15 elemental ratios.

\begin{tabular}{|c|c|c|c|c|c|c|}
\hline & M 15S1 & $\sigma$ & $N_{\text {lines }}$ & M 15S3 & $\sigma$ & $N_{\text {lines }}$ \\
\hline$[\mathrm{Ba} \mathrm{II} / \mathrm{Fe} \mathrm{I}]$ & -0.15 & 0.03 & 4 & 0.29 & 0.06 & 4 \\
\hline$[\mathrm{Ca} \mathrm{I} / \mathrm{Fe} \mathrm{I}]$ & 0.32 & 0.03 & 7 & 0.37 & 0.03 & 7 \\
\hline$[\mathrm{Cr} \mathrm{I} / \mathrm{Fe} \mathrm{I}]$ & -0.43 & 0.05 & 2 & -0.33 & 0.05 & 2 \\
\hline$[\mathrm{Eu} \mathrm{II} / \mathrm{Fe} \mathrm{I}]$ & 0.30 & 0.20 & 1 & 0.65 & 0.10 & 1 \\
\hline$[\mathrm{Fe} \mathrm{I} / \mathrm{H}]$ & -2.36 & 0.02 & 55 & -2.41 & 0.02 & 52 \\
\hline$[\mathrm{Fe} \mathrm{II} / \mathrm{H}]$ & -2.37 & 0.03 & 12 & -2.35 & 0.04 & 12 \\
\hline$[\mathrm{La} \mathrm{II} / \mathrm{Fe} \mathrm{I}]$ & 0.09 & 0.10 & 1 & 0.36 & 0.07 & 3 \\
\hline [Mg I/Fe I] & 0.33 & 0.07 & 3 & 0.28 & 0.07 & 3 \\
\hline$[\mathrm{Mn} \mathrm{I} / \mathrm{Fe} \mathrm{I}]$ & -0.59 & 0.05 & 5 & -0.38 & 0.07 & 6 \\
\hline$[\mathrm{Na} \mathrm{I} / \mathrm{Fe} \mathrm{I}]$ & 0.03 & 0.14 & 2 & 0.68 & 0.11 & 2 \\
\hline$[\mathrm{Nd} \mathrm{II} / \mathrm{Fe} \mathrm{I}]$ & -0.14 & 0.12 & 1 & 0.30 & 0.04 & 2 \\
\hline$[\mathrm{Ni} \mathrm{I} / \mathrm{Fe} \mathrm{I}]$ & 0.07 & 0.06 & 3 & 0.11 & 0.06 & 3 \\
\hline$[\mathrm{O} \mathrm{I} / \mathrm{Fe} \mathrm{I}]$ & 0.04 & 0.10 & 1 & & & 0 \\
\hline [Ti I/Fe I] & -0.08 & 0.09 & 5 & -0.06 & 0.07 & 5 \\
\hline [Ti II/Fe I] & 0.13 & 0.05 & 5 & 0.16 & 0.07 & 5 \\
\hline [Y II/Fe I] & -0.42 & 0.02 & 4 & -0.20 & 0.04 & 3 \\
\hline \multirow[t]{2}{*}[\mathrm{Zn}\mathrm{I}/\mathrm{Fe}\mathrm{I}]{} & 0.03 & 0.05 & 1 & 0.15 & 0.09 & 1 \\
\hline & M 15S6 & $\sigma$ & $N_{\text {lines }}$ & M 15S7 & $\sigma$ & $N_{\text {lines }}$ \\
\hline$[\mathrm{Ba} \mathrm{II} / \mathrm{Fe} \mathrm{I}]$ & 0.39 & 0.04 & 4 & -0.20 & 0.06 & 4 \\
\hline$[\mathrm{Ca} \mathrm{I} / \mathrm{Fe} \mathrm{I}]$ & 0.33 & 0.03 & 6 & 0.41 & 0.03 & 7 \\
\hline$[\mathrm{Cr} \mathrm{I} / \mathrm{Fe} \mathrm{I}]$ & -0.39 & 0.06 & 2 & -0.39 & 0.04 & 2 \\
\hline$[\mathrm{Eu} \mathrm{II} / \mathrm{Fe} \mathrm{I}]$ & 0.81 & 0.10 & 1 & 0.41 & 0.10 & 1 \\
\hline$[\mathrm{Fe} \mathrm{I} / \mathrm{H}]$ & -2.32 & 0.03 & 56 & -2.47 & 0.04 & 49 \\
\hline$[\mathrm{Fe} \mathrm{II} / \mathrm{H}]$ & -2.35 & 0.06 & 13 & -2.50 & 0.04 & 11 \\
\hline$[\mathrm{La} \mathrm{II} / \mathrm{Fe} \mathrm{I}]$ & 0.37 & 0.12 & 2 & 0.09 & 0.18 & 2 \\
\hline$[\mathrm{Mg} \mathrm{I} / \mathrm{Fe} \mathrm{I}]$ & -0.15 & 0.07 & 3 & 0.43 & 0.07 & 3 \\
\hline$[\mathrm{Mn} \mathrm{I} / \mathrm{Fe} \mathrm{I}]$ & -0.41 & 0.06 & 5 & -0.45 & 0.20 & 1 \\
\hline$[\mathrm{Na} \mathrm{I} / \mathrm{Fe} \mathrm{I}]$ & 0.59 & 0.14 & 2 & 0.08 & 0.12 & 2 \\
\hline$[\mathrm{Nd} \mathrm{II} / \mathrm{Fe} \mathrm{I}]$ & 0.28 & 0.03 & 2 & -0.23 & 0.16 & 1 \\
\hline$[\mathrm{Ni} \mathrm{I} / \mathrm{Fe} \mathrm{I}]$ & 0.06 & 0.06 & 3 & 0.00 & 0.06 & 3 \\
\hline$[\mathrm{O} \mathrm{I} / \mathrm{Fe} \mathrm{I}]$ & -0.05 & 0.10 & 1 & 0.15 & 0.10 & 1 \\
\hline$[\mathrm{Ti} \mathrm{I} / \mathrm{Fe} \mathrm{I}]$ & -0.09 & 0.07 & 6 & -0.04 & 0.11 & 4 \\
\hline [Ti II/Fe I] & 0.14 & 0.05 & 5 & 0.07 & 0.07 & 5 \\
\hline [Y II/Fe I] & -0.15 & 0.04 & 3 & -0.37 & 0.04 & 4 \\
\hline$[\mathrm{Zn} \mathrm{I} / \mathrm{Fe} \mathrm{I}]$ & 0.02 & 0.10 & 1 & 0.04 & 0.08 & 1 \\
\hline
\end{tabular}

